\title{
Macromolecular crowding and membrane binding proteins: the case of phospholipase $A_{1}$
}

Yuzhang Wei ${ }^{1}$, Isabel Mayoral-Delgado ${ }^{1}$, Nicolas A. Stewart ${ }^{1}$, Marcus K. Dymond ${ }^{1 *}$

${ }^{1}$ Division of Chemistry, School of Pharmacy and Biological Sciences, University of Brighton, BN2 $4 G L$

*Author for correspondence: M.Dymond@brighton.ac.uk

Keywords: Phospholipase $A_{1}$, macromolecular crowding, 3D-printing, membrane binding, equilibrium kinetics

\footnotetext{
Abstract

Cells contain high levels of macromolecular crowding; understanding how macromolecular crowding impacts the behaviour of biological systems can give new insights into biological phenomena and disease pathologies. In this study, we assess the effect of macromolecular crowding on the catalytic activity of the biomembrane binding protein phospholipase $A_{1}\left(P L A_{1}\right)$. Using 3D-printed equilibrium dialysis chambers we show that macromolecular crowding increases the binding of PLA 1 to lipid vesicles. However, using a mass spectrometry assay of the hydrolysis of 1,2-dioleoyl-sn-glycero-3-phosphocholine (DOPC) by PLA ${ }_{1}$ we surprisingly find that macromolecular crowding decreases the reaction rate and causes early cessation of the catalytic activity of PLA 1 . Using kinetic equilibrium modelling, we are able to estimate the effect of macromolecular crowding on the association and dissociation rate constants for PLA $\mathrm{A}_{1}$ binding to the lipid vesicles. These data, coupled with particle sizing measurements enable us to construct a model to explain the early cessation of catalytic activity of PLA $_{1}$ with increasing levels of macromolecular crowding. This model suggests that compositional changes in the membrane, due to $\mathrm{PLA}_{1}$ action, lead to the formation of larger vesicles, which deactivate the protein. This process is more rapid in the presence of macromolecular crowding agents, suggesting that a more detailed understanding of the effects of macromolecular crowding on membrane dynamics is required to understand membrane interacting proteins in
} 
macromolecularly crowded environments. The implications of this discovery are significant given the wide range of roles of membrane fusion and fission in neurocognitive processes and the failure of these processes in neurodegenerative diseases.

\subsection{Introduction}

Macromolecular crowding describes the phenomenon that occurs when solutions contain large numbers of macromolecules. These macromolecules occupy space, which other molecules are excluded from (the excluded volume effect), and reduce the thermodynamic activity of water [1]. Interest in macromolecular crowding stems from the observation that the interior of cells is a crowded macromolecular environment. Hence the addition of crowding agents to enzyme assays provides a way to mimic these crowding effects in vitro. In general, crowded macromolecular conditions favour the folded state of proteins and increase aggregation and binding processes, which may play a role in the formation of amyloid plaques in Alzheimer's disease and other age-related neurodegenerative diseases [2]. Exosomes have also been implicated in neurodegenerative diseases and it has been noted that phospholipid metabolising enzymes such as phospholipases, which play a role in exosome formation, show atypical behaviour in Alzheimer's disease $[3,4]$. In this work we assess the impact of macromolecular crowding on phospholipase $\mathrm{A} 1\left(\mathrm{PLA}_{1}\right)$.

\subsection{Macromolecular crowding effects on proteins}

Theory predicts that macromolecular crowding will impact proteins in several ways, the most immediate being through the reduction of molecular diffusion coefficients $[5,6]$. When protein-protein and protein-membrane interactions are important, reduced diffusion coefficients mean that two chemical entities will take longer to collide and associate. On the other hand, two chemical entities recently associated also have a higher probability of colliding again, thus an increase in the equilibrium association constant is often observed, such as DNA polymerase binding to DNA [7]. It should also be noted that in a more complex process, where 
an orientational component to the kinetics of association exists, an increase in equilibrium binding may not be observed [8].

Macromolecular crowding agents increase the effective concentrations of reagents in solution. This occurs through the excluded volume effect and by decreasing the thermodynamic activity of water. Both processes increase the equilibrium association constants of proteins with surfaces, or proteins with their substrates, typically causing accelerated reaction rates to be observed $[9,10]$ under crowded conditions. Conformational changes in proteins $[1,11,12]$ are also frequently induced by macromolecular crowding agents, with theory predicting that the stability of the folded state of the protein will be increased by crowding. This originates from the observation that unfolded proteins have a larger volume than folded proteins and hence the excluded volume effect of molecular crowding agents favours the folded state. It should be noted that theory predicts the stabilising effect of crowding agents to be much greater than the values typically observed in experimental systems [13].

\subsection{PLA 1 : structural and interfacial effects on catalytic activity}

Here we report the impact of macromolecular crowding on the activity of a protein engineered form of PLA 1 , EC 3.1.1.32, from a Thermomyces lanuginosus host, (Lecitase Ultra) on 1,2-dioleoyl-sn-glycero-3-phosphatidylcholine (DOPC). PLA 1 , is one of the least studied phospholipases [14], which catalyses the hydrolysis of the 1-acyl chain of phospholipids to give a fatty acid (oleic acid (OA), in this study) and a 2-lyso phospholipid as products [15]. This particular variant of $\mathrm{PLA}_{1}$ contains 339 amino acids and is a hybrid of $\mathrm{PLA}_{1}$ from $T$. lanuginosus (TII), residues 1-284, and Fusarium oxysporum lipase (FoL) (residues 285-339), but containing point mutations (compared to TII) of Gly113Ala, Asp118Trp and Glu121Lys. Lecitase Ultra has a molar mass of $35 \mathrm{kDa}$ and shows lower activity than many PLA $\mathrm{A}_{1}$ variants in nature. For example, the commercial enzyme preparation of Lecitase Ultra $(65 \mathrm{mg} / \mathrm{ml})$ has activity of $32 \mathrm{units} / \mathrm{mg}$ protein, which is lower than lipase from porcine pancreas (125 units/ mg) and Amano lipase PS (177 units/mg) [16]. Maximal activity is observed at $\mathrm{pH} 8.5$ with around two thirds maximal activity observed at $\mathrm{pH}$ 7.0. The enzyme shows high activity over the temperature range from 
20 to $50^{\circ} \mathrm{C}$, with maximal activity from 20 to $30^{\circ} \mathrm{C}$ [16] and a rapid loss of activity from 60 to $70^{\circ} \mathrm{C}$. Lecitase Ultra appears to require high concentrations of $\mathrm{CaCl}_{2}$ for optimal activity, with Mishra et al. [16] reporting that $25 \mathrm{mM} \mathrm{CaCl}_{2}$ enhances activity.

$\mathrm{PLA}_{1}$ exhibits catalytic activity towards phospholipids at an interface between the aqueous environment and the lipid aggregate. Typically vesicles are studied, however, the action of phospholipases on a range of other lipid aggregates, monolayer interfaces [17] and lyotropic liquid crystal phases has also been investigated [18-21]. These studies show that the interfacial properties of the aggregate are just as important as substrate specificity in determining overall lipase activity. For example, Wang et al. [22] show that the activity of Lecitase Ultra is highly dependent on the packing of lipid headgroups, when surface pressure is applied to the lipids in a Langmuir trough. Under such conditions, activity against DOPC ranges from negligible, at a surface pressure of $10 \mathrm{mNm}^{-1}$, to $200 \times 10^{-11}\left(\right.$ moles $\left.\mathrm{cm}^{-2} \mathrm{~min}^{-1} \mathrm{ml}^{-1}\right)$ at a surface pressure of $20 \mathrm{mNm}^{-1}$. These observations are important since bilayer membranes with different chemical compositions can have dramatically different biophysical properties, which can in turn impact the activity of membrane interacting proteins.

For example, some lipid compositions form homogenous mixtures while others, notably PC and cholesterol mixtures, form laterally segregated domains in the membrane [23]. In addition, the different curvature preferences of lipids gives rise to different quantities of stored curvature elastic energy in the bilayer membrane [24], through the frustration of lipid spontaneous curvatures, and the effect of Gaussian curvature on factors such as head group packing. Work on phospholipase $A_{2}$, for example, suggests this class of enzyme senses the mechanical properties of lipid monolayers/ bilayers [27] like membrane curvature elastic stress [26].

X-ray crystallography has shown that a 'lid'-like structure present in TII phospholipase [28], is highly preserved in other variants of the enzyme [15]. This lid confers maximal activity when in the open state; lipids activate the enzyme by opening the lid and ionic strength closes the lid, deactivating the enzyme. It is also possible that the biophysical properties of lipid membranes like lateral phase separation and stored curvature elastic stress, which are 
important for other membrane binding proteins $[25,26]$, might cause structural changes in PLA that accompany changes in catalytic activity and lid opening, although this is yet to be elucidated. Furthermore, since $\mathrm{PLA}_{1}$ generates lysophosphatidylcholine and fatty acids, significant changes in the biophysical properties of the bilayer interface probably occur over the

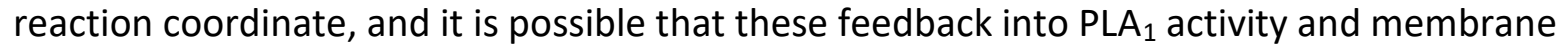
binding. For example, PC/OA lipid mixtures have a spontaneous curvature that prefers tightly curved inverted structures, whilst PC forms flat lamellar structures [29]. Although, it is worth noting that 1:1 OA, lyso-oleoylphosphatidylcholine mixtures still form stable vesicles [30]. The presence of cations, such as calcium in this study, can also have significant effects on the spontaneous curvatures of anionic lipids [31] and cause lateral phase separation in vesicle systems containing anionic lipids, like phosphatidylserine and phosphatidic acid [32,33]. Similarly, the presence of anionic lipids in a membrane with calcium cations in the media can cause membrane fusion events and promote vesicle aggregation [34]. These observations suggest that the production of fatty acids by phospholipases, especially calcium dependent phospholipases, might also modify the lipid membrane interface by causing lateral phase separation of the fatty acids. This adds a considerable level of complexity to studying interfacial enzymes such as PLA ${ }_{1}$, which might sense curvature elastic stress, packing defects or OA rich domains in the membrane caused by lateral phase separation.

\subsection{Kinetic models of phospholipase activity}

Kinetic models have been developed to quantify the catalytic properties of phospholipases at interfaces. These surface dilution models $[35,36]$ rationalise the activity of phospholipases in two steps. The first is bulk solution behaviour where the enzyme associates with the surface of a vesicle (or micelle), Scheme 1. The second is an interfacial step (Scheme 2 ), where the chemical activity of the phospholipase occurs on the aggregate surface.

$$
P L A+V \underset{k_{-1}}{\stackrel{k_{1}}{\rightleftharpoons}} P L A V \quad \text { Scheme } 1
$$


Where PLA is a generic phospholipase, $V$ is a binding site on the lipid vesicle and $k_{1} / k_{-1}$ are the rate constants for the association and dissociation of the enzyme with the vesicle.

$$
\begin{aligned}
& k_{2} \quad k_{3} \\
& P L A V+L \rightleftharpoons P L A V L \rightleftharpoons P L A V+\text { products } \\
& k_{-2} \quad k_{-3}
\end{aligned}
$$

Scheme 2

Scheme 2 describes the chemical reaction where the surface-bound enzyme (PLAV) collides with a lipid $(L)$ to form a complex where $L$ is hydrolysed to give the reaction products and PLA bound to the vesicle surface. Several groups have modelled this process as a 2dimensional Michaelis-Menten scheme $[35,37,38]$, where reactions catalysed by PLA occur through two mechanisms. The first is a 'scooting' mechanism, where the phospholipase is irreversibly bound and only moves on a single vesicle surface. The second is a 'hopping' mechanism, where PLA reversibly moves between aqueous solution and vesicles [38]. This type of study is usually designed so that lipid vesicles are present in large excess, 'irreversibly' binding the protein to the membrane to determine kinetic parameters in the scooting mode [39]. This approach is not easily adapted to macromolecular crowding studies, where it is desirable to have the phospholipase 'reversibly' bound to the membrane to enable macromolecular crowding effects on the binding equilibria of proteins to vesicles to be observable.

\subsection{Aims of the study}

In this study we look at the effect of the macromolecular crowding agent Ficoll 400 [1] on the activity of PLA $A_{1}$. Ficolls are highly branched polysaccharides comprised of sucrose and epichlorohydrin building blocks $[40,41]$. The advantage of using Ficoll in protein-crowding studies is that Ficolls do not interact chemically with proteins $[40,42]$. Furthermore, low concentrations (up to $5 \mathrm{wt} \%$ ) of Ficoll have little effect on the bulk viscosity of aqueous 
solutions, therefore crowding effects can be easily disentangled from bulk viscosity effects. Phospholipases are one of a number of lipid biosynthetic enzymes involved in lipid homeostasis [43-46] and understanding this cellular mechanism is one aspect of this work. In particular, since many charged lipids interact with divalent cations, which can change lipid spontaneous curvatures and stored elastic energy in membranes [31], we carried out our studies at relatively high calcium concentrations. This approach is well suited to Lecitase ultra which is activated by $\mathrm{CaCl}_{2}[16]$.

Finally, the increased use of phospholipids in drug and gene delivery applications [4750] makes it important to understand the stability of such drug delivery complexes in vivo. A critical step in this process is the development of new methodology to quantify the activity of phospholipases $[51,52]$ and the development of protocols to enable studies on interfacially active proteins to be performed under conditions of macromolecular crowding. By combining kinetic equilibrium modelling and 3D-printed reactionware, we herein present simple methodology that enables the different effects of macromolecular crowding on interfacial proteins to be disentangled. 


\subsection{Experimental}

DOPC was purchased from Avanti Polar Lipids, USA. Ficoll PM 400 was purchased from GE Healthcare, UK. Sodium chloride, calcium chloride, iron (III) chloride. $6 \mathrm{H}_{2} \mathrm{O}$ and ammoniumthiocyanate were purchased from Acros Organics, UK. Trizma preset crystals (pH 7.2), phospholipase $A_{1}$ (Lecitase Ultra from T. lanuginosus), Coomassie Brilliant Blue G-250 and chloroform were purchased from Sigma Aldrich, UK. All chemicals were used without further purification.

Freeze-drying, particle sizing and 3D-printing were carried out using a CHRIST ${ }^{\circledR}$ Alpha 2-4 freezedryer, a ZetasizierNano - ZS90 (Malvern Instruments) and Replicator 2X Experimental 3Dprinter (Makerbot) equipped with polylactic acid filament (EasyFil PLA, Form Futura), respectively. Mass spectrometry was performed using an Esquire HCT Plus (Bruker Daltonics) fitted with an Agilent 1200 binary high performance liquid chromatography (HPLC) system for sample fractionation. UV visible spectroscopy was performed on a dual beam Shimadzu Corporation UV-2401PC spectrometer equipped with a single monochromator. BRAND ${ }^{\circledR}$ disposable polystyrene cuvettes (Sigma Aldrich UK) were used to record all spectra.

\subsection{Preparation of assay buffers}

Four buffer systems were used in this work containing 0 (standard uncrowded buffer), 0.5, 1 or 2 wt \% Ficoll PM 400 in Trizma (50 mM), sodium chloride (40 mM) and calcium chloride (100 $\mathrm{mM}$ ) at pH 7.2. Crowded buffers containing Ficoll PM 400 were prepared by slowly adding Ficoll powder to the standard uncrowded assay buffer, followed by stirring and gentle heating in a water bath.

\subsection{Preparation of unilamellar vesicles}

Unilamellar vesicles, at a final concentration of $15 \mathrm{mM}$ (total lipid), were prepared from $100 \mu \mathrm{l}$ $(2.54 \mu \mathrm{mol})$ of stock DOPC solution $(20 \mathrm{mg} / \mathrm{ml})$, deposited in a $0.5 \mathrm{ml}$ microcentrifuge tube. Chloroform was removed using a stream of argon gas and the dried lipids samples were left in 
vacuo for 2 hours. Samples were hydrated with $200 \mu$ of deionized water, frozen at $-80^{\circ} \mathrm{C}$ for 1 hour and lyophilised, prior to the addition of $169.3 \mu$ l of assay buffer. Samples were vortexed for 10 minutes followed by 20 minutes rest, sonicated for 20 minutes and rested for a further 30 minutes at room temperature. Three freeze-thaw cycles were carried out, where samples were frozen at $-80^{\circ} \mathrm{C}$ followed by 20 minutes rest at room temperature. After the final resting phase, samples were stored at $-20^{\circ} \mathrm{C}$. Before use, vesicles were defrosted at room temperature, sonicated for 5 minutes and then rested for 30 minutes. Crowded vesicles were prepared using the same procedure but lipids were resuspended in Ficoll PM 400 buffers of $0.5,1$ or 2 wt \% as required. This methodology gave vesicles with an average diameter of 100 to $300 \mathrm{~nm}$ and a typical polydispersity index in the range of 0.8 to 1 .

\subsection{Mass spectrometric assay of PLA 1 activity}

The activity of PLA 1 was assayed in uncrowded or crowded buffer systems in a volume of $1 \mathrm{ml}$ and final PLA 1 and DOPC conditions of $0.35 \mathrm{mg} / \mathrm{ml}$ and $0.2 \mathrm{mM}$, respectively. Assays were performed in triplicate and measurements were made at 0, 10, 40 and 70 minutes. Reactions were terminated by decanting $800 \mu \mathrm{L}$ of the reaction mixture into a chloroform, methanol and water mixture (2 $\mathrm{ml}: 2 \mathrm{ml}: 1 \mathrm{ml})$, as per the Bligh-Dyer total lipid extraction method [53]. After centrifugation (500 g, $5 \mathrm{~min}$ ), the lower organic layer was separated and dried in vacuo. Samples were resuspended in a solvent system of water: methanol: butanol (6:3:1) containing $10 \mathrm{mM}$ ammonium acetate and stored in a freezer $\left(-80^{\circ} \mathrm{C}\right)$.

Prior to mass spectrometry analysis, samples $(10 \mu \mathrm{L})$ and standards were separated by HPLC (Agilent 1200 binary) fitted with a C18 reversed phase column (Kinetex $2.6 \mu$ F5 $100 \AA 150$ $\mathrm{mm} \mathrm{L} \times 2.1 \mathrm{~mm}$ diameter with guard column) at $50^{\circ} \mathrm{C}$ using a gradient with a flow rate of 300 $\mu \mathrm{L} / \mathrm{min}$. Gradient conditions for eluent $\mathrm{A}\left(\mathrm{ddH}_{2} \mathrm{O}, 0.1 \%\right.$ formic acid, $10 \mathrm{mM}$ ammonium acetate) and eluent B (acetonitrile:2-propanol (5:2), 0.1\% formic acid, $10 \mathrm{mM}$ ammonium acetate) were as follows; $15 \%$ B for $2.5 \mathrm{~min}$, increased to $100 \%$ B over one min, held at $100 \%$ B for 6 min, 
decreased back to $15 \%$ B over 30 seconds and equilibrated at $15 \%$ B for 3 min. Total runtime and data acquisition time was $13 \mathrm{~min}$.

After separation, samples were analysed by electrospray ionisation using an ion trap mass spectrometer (Esquire HCT Plus, Bruker Daltonics) operating in the positive mode. A spectral range from $500-800 \mathrm{~m} / \mathrm{z}$ was recorded. Analysis parameters were as follows; ultra scan mode, target mass 790, nebulizer gas at 45 psi, dry gas at $7 \mathrm{~L} / \mathrm{min}$, dry temp. $300^{\circ} \mathrm{C}$, Cap. voltage $-4000 \mathrm{~V}$, Skimmer at $40 \mathrm{~V}$, Cap. exit at $150 \mathrm{~V}$. Maximum acquisition time was set to 200 ms with an ICC target of 200,000 . Two peaks corresponding to DOPC $[\mathrm{M}+\mathrm{H}]^{+}$and $[\mathrm{M}+\mathrm{Na}]^{+}(786.7$ $\mathrm{m} / \mathrm{z}$ and $808.6 \mathrm{~m} / \mathrm{z}$, respectively) were detected. A calibration curve was constructed using the $[\mathrm{M}+\mathrm{H}]^{+}$ion from known concentrations of DOPC, and the concentration of DOPC in the reaction samples was calculated using QuantAnalysis Software (V 3.3, Build 149, Bruker Daltonics). Samples for the calibration curve were run, followed by samples in $0,0.5,1$ and 2 wt\% Ficoll 400 at the four studied times. PLA 1 activity was determined by quantifying the amount of DOPC remaining in the samples.

2.4 3D-printed equilibrium dialysis chamber: measuring non-specific binding of $P L A_{1}$ and DOPC to 3D-prints

3D-printed equilibrium dialysis chambers were assembled using polylactic acid filament at $50 \%$ infill and the standard Makerbot polylactic acid print settings, summarised previously [54]. Controls were performed to determine the extent of non-specific binding of PLA $A_{1}$ and DOPC to the 3D-printed material. For these control studies DOPC was quantified using the Stewart assay [55] and PLA 1 was quantified using the Bradford assay [56]. Standard curves were determined for each of the crowded and uncrowded buffer systems for both the assays see Figures S1 to S8, as discussed in sections S1 and S2. Section S3, Figure S9 shows the 3D-printed sample tubes used to determine non-specific binding of PLA $\mathrm{A}_{1}$ and DOPC to the 3D-printed material, Figures S10 and S11 show time-dependent trends of non-specific binding. 


\subsection{D-printed equilibrium dialysis chamber: control studies}

Equilibrium dialysis systems were 3D-printed to contain two chambers, A and B, separated by a dialysis membrane with $50 \mathrm{kDa}$ molecular weight cut-off (Spectra/Por ${ }^{\circledR}$ Dialysis membrane tubing, Biotech CE Spectrum labs). The dialysis membrane was washed according to the manufacturer's instructions and the two halves of the chamber were clamped into place around the membrane using a G-clamp. In chamber A, $1 \mathrm{ml}$ of buffer and either PLA (typical starting concentration $0.9 \mathrm{mg} / \mathrm{ml})$ or DOPC vesicles $(1.5 \mathrm{mM})$ was added. In chamber B, $1 \mathrm{ml}$ of buffer was added and the device was covered with parafilm. The passage of DOPC vesicles or $\mathrm{PLA}_{1}$ through the membrane was monitored by removing $0.1 \mathrm{ml}$ aliquots from both chambers $\mathrm{A}$ and B at defined time intervals. See Section S4, Figures S12 and S13 for further information.

To quantify DOPC, $0.4 \mathrm{ml}$ of buffer was added to $0.1 \mathrm{ml}$ of samples to enable direct comparison with the calibration curves. Samples were freeze-dried and $2 \mathrm{ml}$ of chloroform and $2 \mathrm{ml}$ of ammonium ferrothiocyanate reagent were added as per the Stewart assay, see section S2. $0.1 \mathrm{ml}$ protein samples were added to $3 \mathrm{ml}$ of Bradford reagent as per the Bradford assay (see section S1). Studies were performed in triplicate in crowded and uncrowded buffers.

\subsection{D-printed equilibrium dialysis chambers: measurement of $P L A_{1}$ : vesicle equilibrium} association constants

3D-printed equilibrium dialysis chambers were pre-equilibrated by adding $1 \mathrm{ml}$ of buffer and $\operatorname{PLA}_{1}(0.9 \mathrm{mg} / \mathrm{ml})$ to chamber $A$ and $1 \mathrm{ml}$ of buffer to chamber $B$. This mixture was left for 2 hours to equilibrate between chambers $A$ and $B$ (time determined from the control study performed in Section 2.5). After this time, $0.5 \mathrm{ml}$ of solution was removed from chambers $A$ and $\mathrm{B}$, and $0.5 \mathrm{ml}$ of vesicles $(1.5 \mathrm{mM})$ was added to chamber $B$ and $0.5 \mathrm{ml}$ of buffer was added to chamber A, before the device was covered with parafilm to prevent evaporation. This approach was used to obtain a PLA 1 and DOPC ratio comparable to that used in the mass spectroscopy and dynamic light scattering studies i.e in the range of 0.2 to $0.35 \mathrm{mg} \mathrm{ml} \mathrm{PLA}$ and 0.15 to 0.2 $\mathrm{mM}$ DOPC vesicles. Although it should be noted the non-specific binding of PLA 1 in the 
equilibrium dialysis experiment makes this difficult to control in a precise way. The concentration of $\mathrm{PLA}_{1}$ was monitored with respect to time by removing aliquots of $0.1 \mathrm{ml}$ from both chambers $A$ and B, adding to $3 \mathrm{ml}$ of Bradford reagent (see Section 2.5). Studies were performed in triplicate in both crowded and uncrowded buffers.

\subsection{Differential scanning calorimetry studies of $P L A_{1}$ in Ficoll 400}

Differential scanning calorimetry (DSC) studies of PLA 1 in Ficoll 400 were performed on DSC Q2000 (TA instruments) using a modulated DSC setting $\left(0.5^{\circ} \mathrm{C}\right.$ modulation temperature over 100 seconds), equipped with nitrogen as a purge gas at a flow rate of $10 \mathrm{ml} / \mathrm{min}$. Samples of $\mathrm{PLA}_{1}$ were prepared at a final concentration of $31 \mathrm{mg} / \mathrm{ml}$ in $0.1 \mathrm{M}$ phosphate buffer or 50 mM PIPES containing $100 \mathrm{mM} \mathrm{CaCl}_{2}$ and $40 \mathrm{mM} \mathrm{NaCl}$ (pH 7.2) and 0, 0.5, 1, 2, 4 and 8 wt \% Ficoll 400 as appropriate. $10 \mu$ volumes of each sample were weighed, hermetically sealed in TZero aluminium pans (TA instruments) and studied from 0 to $100^{\circ} \mathrm{C}$ at a heating rate of 2 degrees per minute, with an empty hermetically sealed pan as reference. Studies were performed in phosphate and PIPES buffers due to the thermal susceptibility of Tris buffer. Bovine serum albumin $(87 \mathrm{mg} / \mathrm{ml} 0.1 \mathrm{M}$ phosphate buffer $\mathrm{pH} 7.2)$ was run as a standard.

\subsection{Dynamic light scattering studies of $P L A_{1}$ reacting with DOPC vesicles}

For each experiment, $30 \mu \mathrm{l}$ of vesicles $(15 \mathrm{mM})$, prepared according to Section 2.2, were added to $3 \mathrm{ml}$ of buffer in a disposable plastic cuvette, $10 \mu \mathrm{l}$ of PLA $(65 \mathrm{mg} / \mathrm{ml})$ in water were added to this solution, and the components were mixed by inversion to give a final concentrations of $0.2 \mathrm{mg} / \mathrm{ml} \mathrm{PLA} 1$ and $0.15 \mathrm{mM}$ DOPC vesicles. Measurements were performed in triplicate at 10 minute intervals at an angle of $90^{\circ}$ and at $25^{\circ} \mathrm{C}$. Control studies were

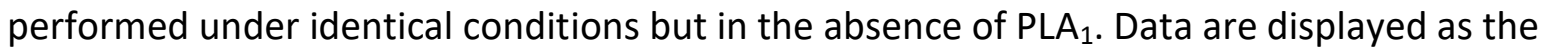
mean average vesicle diameter of three different vesicle samples, error bars are the standard deviation of the mean. 


\subsection{Equilibrium association modelling of $P L A_{1}$ in a 3D-printed equilibrium dialysis chamber}

Equilibrium association models were constructed using the COPASI [57] biochemical network simulator (version 4.11, build 65). Equilibrium dialysis processes were modelled as a 2 compartment system, with the same properties as the 3D-printed equilibrium dialysis device (i.e. $2 \times 1 \mathrm{ml}$ chambers), using mass action kinetics. Equilibrium steps E1 and E2 (see Scheme 3) were modelled, using a starting concentration of $\mathrm{PLA}_{1}$ binding sites $(\mathrm{V})$ of $1.6 \times 10^{-5} \mathrm{M}$. The starting concentrations of $\mathrm{PLA}_{1 \mathrm{~A}}$ and $\mathrm{PLA}_{1 \mathrm{~B}}$ in chambers $\mathrm{A}$ and $\mathrm{B}$ were initially equal and determined from the results presented. Actual values used were $7.1 \times 10^{-6}, 7.5 \times 10^{-6}, 7.6 \times 10^{-6}$ and $8.3 \times 10^{-6} \mathrm{M}$, at $0,0.5,1$ and $2 \mathrm{wt} \%$ Ficoll 400 . Section S4.3 shows the differential equations used by COPASI in the modelling.

Optimal curve fitting was achieved by manually adjusting the forward and backward rate constants $\left(k_{a}\right.$ and $\left.k_{b}\right)$ to model the broad timescale of the dialysis process, whilst maintaining the experimentally determined equilibrium association constants of PLA $\mathrm{A}_{1}$ with the vesicles. Subsequently, manual optimisation of the forward and reverse association rate constants of PLA 1 with the vesicles $\left(k_{1}\right.$ and $\left.k_{-1}\right)$ was carried out to achieve the best data fit. This process was initially performed on the $0 \mathrm{wt} \%$ Ficoll 400 system. Once optimised, the $0.5,1$ and 2 wt \% Ficoll systems were modelled by applying a bulk correction factor to $k_{a}, k_{b}, k_{1}$ and $k_{-1}$, which treats the process as diffusion controlled, as discussed in the results and discussion.

\subsection{Equilibrium association modelling of the reaction kinetics of PLA $A_{1}$ with DOPC vesicles}

$\mathrm{PLA}_{1}$ catalysed reactions of the hydrolysis of DOPC were modelled in a single compartment using equilibrium steps E2 to E4 (Scheme 3). Starting concentrations of PLA $A_{1 B}, V$ and DOPC were $1.5 \times 10^{-5} \mathrm{M}, 0.4 \times 10^{-5} \mathrm{M}$ and $2 \times 10^{-4} \mathrm{M}$ respectively. Values of $\mathrm{k}_{1}$ and $\mathrm{k}_{-1}$ at different wt \% of Ficoll 400 were obtained from the results of equilibrium association model of the dialysis chamber (Section 2.9). Curve fitting was achieved by manually adjusting the forward and backward rate constants $\left(\mathrm{k}_{2}, \mathrm{k}_{-2}\right.$ and $\left.\mathrm{k}_{3}, \mathrm{k}_{-3}\right)$ to model the broad timescale of the reaction 
process in line with the model proposed in the results and discussion. Section S4.3 shows the differential equations used by COPASI in the modelling.

\section{Results}

\subsection{Quantifying membrane binding of PLA $A_{1}$ to vesicles in 3D-printed equilibrium dialysis} chambers

Initially, we set out to quantify the membrane binding of $\mathrm{PLA}_{1}$ to vesicles using a centrifugation technique to separate vesicles and non-membrane bound protein [58]. However this approach failed in the presence of crowding agents, which prevented the vesicles from forming a defined 'pellet' during centrifugation. Therefore, we constructed a 3D-printed equilibrium dialysis system to enable us to quantify the binding of PLA 1 to lipid vesicles. Using this device introduces a further kinetic step to the schemes shown in Scheme 1 and 2, as shown in Scheme 3.

$$
\underset{k_{d}}{\stackrel{\text { E1 }}{k_{a}} P L A_{1 B}}+\underset{k_{1 A}}{\stackrel{\text { E2 }}{k_{1}} P L A_{1 B} V}+\stackrel{\text { E3 }}{\rightleftharpoons} \underset{k_{-1}}{\stackrel{k_{2}}{\rightleftharpoons}} P L A_{1 B} V(D O P C) \stackrel{\text { E4 }}{\stackrel{k_{3}}{\rightleftharpoons}} P L A_{1 B} V+O A+O P C
$$

\section{Scheme 3.}

Where $\mathrm{PLA}_{1 \mathrm{~A}}$ is the $\mathrm{PLA}_{1}$ in chamber $A, \mathrm{PLA}_{1 \mathrm{~B}}$ is the $\mathrm{PLA}_{1}$ in chamber $\mathrm{B}$, not associated with vesicles, and $P L A_{1 B} V$ is $P_{L A}$ bound to vesicles. The terms $k_{a}$ and $k_{d}$ are the forward and reverse rate constants of $\mathrm{PLA}_{1}$ passing through the dialysis membrane (note $\mathrm{PLA}_{1}$ associated with the dialysis membrane (PLA $)$ is assumed to be constant and encompassed in the rate constants $k_{a}$ and $k_{d}$ ). $k_{1}$ and $k_{-1}$ are the association/ dissociation rate constants of PLA $1 B$ 
interacting with the vesicle binding sites $(\mathrm{V}), \mathrm{k}_{2}$ and $\mathrm{k}_{-2}$ are association/ dissociation rate constants of $\mathrm{PLA}_{1 \mathrm{~B}} \mathrm{~V}$ binding to a DOPC monomer and $\mathrm{k}_{3}, \mathrm{k}_{-3}$ are the forward and reverse rate constants of the hydrolysis of DOPC to 1-hydroxy-2-oleoyl-sn-glycero-3-phosphocholine (OPC) and OA. E1 to E4 (Scheme 3) define the 4 equilibrium steps of the process and Figure 1 shows the 3D-printed device used to make measurements.

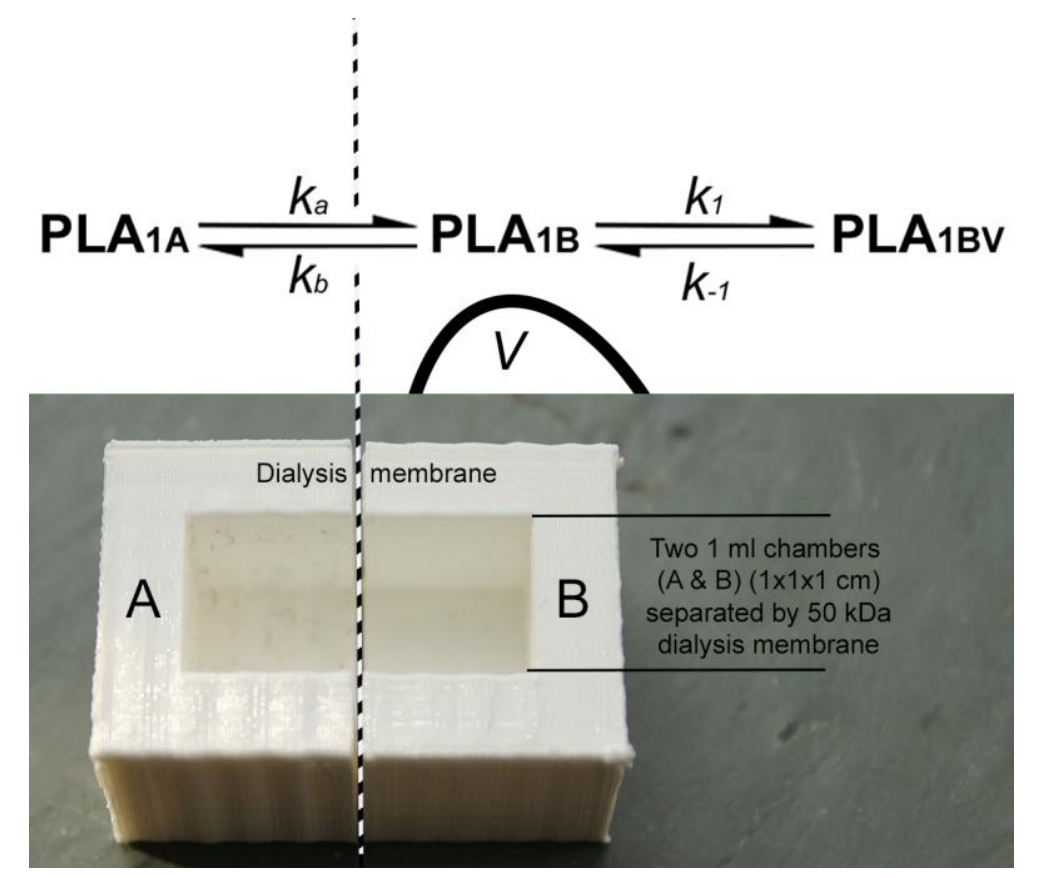

Figure $1 \mathrm{~A}$ 3D-printed equilibrium dialysis system with $50 \mathrm{kDa}$ dialysis membrane for the analysis of PLA $\mathrm{A}_{1}$ binding to lipid vesicles. PLA $(35 \mathrm{kDa}$ ) is placed in chamber $A$ and allowed to equilibrate into chamber $B$, the reaction is initiated by placing vesicles $(V)$ into chamber $B$ and $\left[P L A_{1 A}\right]$ is measured with respect to time. The scheme shows the passage of $P L A_{1}$ molecules through the membrane before binding to the vesicles, steps E1 and E2, Scheme 3.

Control studies confirmed that DOPC vesicles do not cross the dialysis membrane (Figure S12) but PLA 1 does, taking a couple of hours to equilibrate (Figure S13). These studies also showed that PLA binds non-specifically to the polylactic acid filament used to produce the 3D print but DOPC vesicles do not. Full details are shown in supporting information, Sections S3 and S4. 


\subsection{The effect of macromolecular crowding on the equilibrium binding of PLA $A_{1}$ to DOPC vesicles}

Using a protocol to saturate non-specific binding sites with $\mathrm{PLA}_{1}$ before performing measurements, we measured $\left[\mathrm{PLA}_{1 \mathrm{~A}}\right]$ with respect to time, as detailed in Section 2.6. This protocol ensures that $\mathrm{PLA}_{1}$ has equilibrated through the dialysis membrane and is in equal concentration in chambers $A$ and $B$, prior to addition of DOPC vesicles to chamber $B$. The advantage of this approach is that it prevents slow passage of $\mathrm{PLA}_{1}$ through the dialysis membrane, from being the rate determining step in the binding of $\mathrm{PLA}_{1}$ to the vesicles. Figure 2 shows the results of these studies.

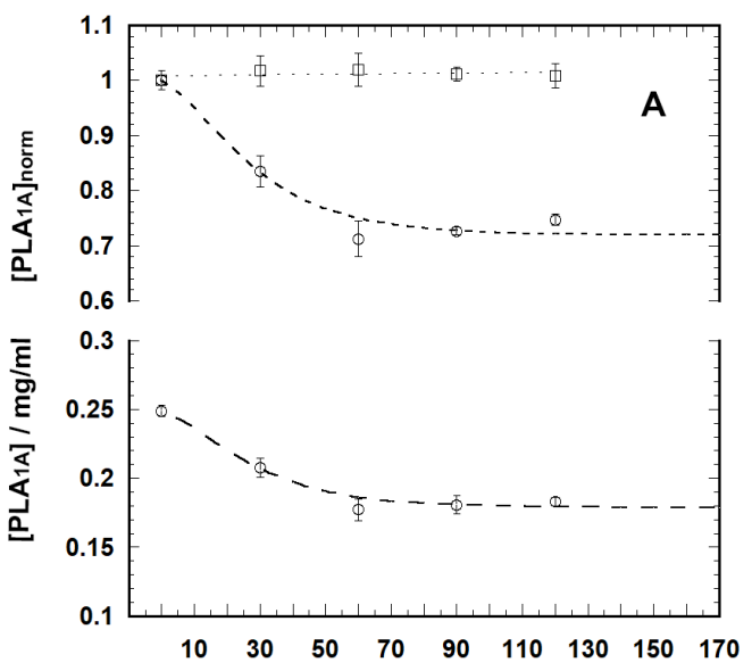

Time / mins

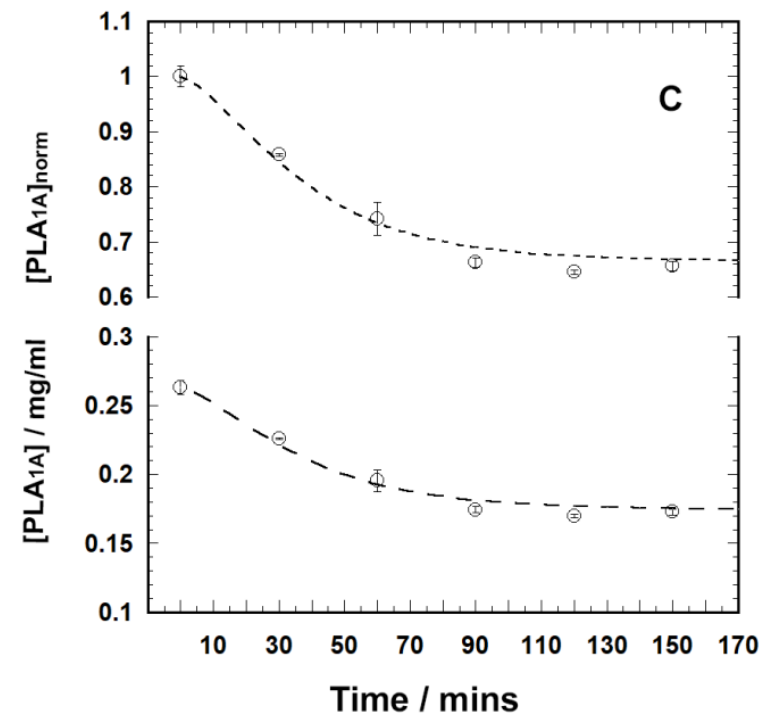

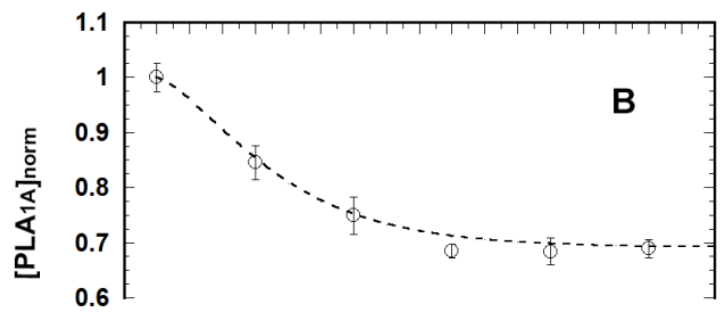

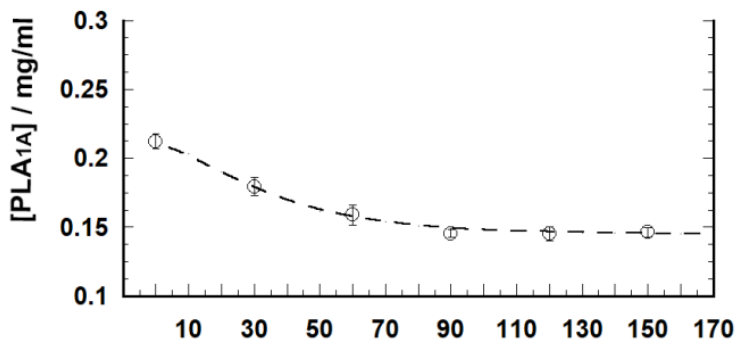

Time / mins
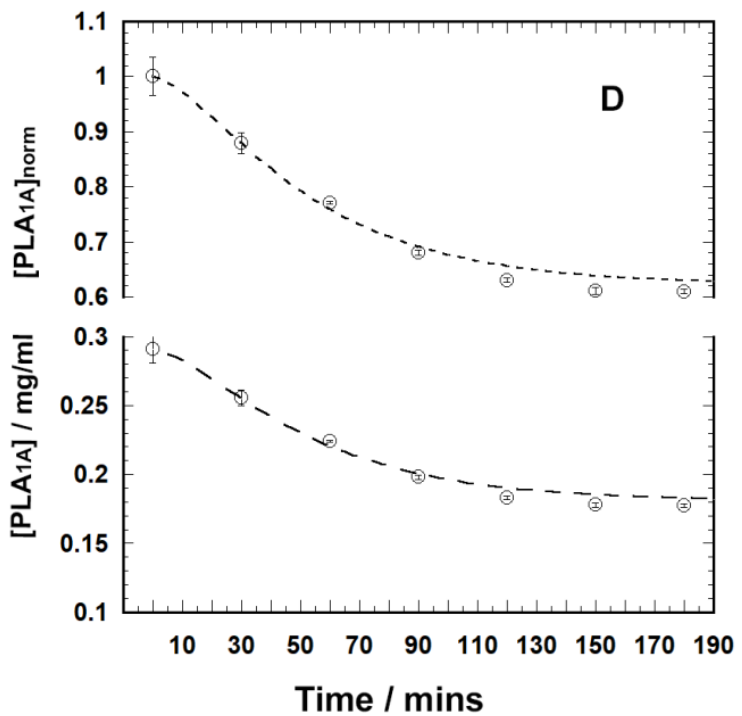
Figure 2 the variation in $\left[\mathrm{PLA}_{1 \mathrm{~A}}\right]$ with respect to time, measured in equilibrium dialysis chambers. Figure $2 \mathrm{~A}$ shows data obtained in 0 wt $\%$ Ficoll 400 , unfilled squares show a control study with no vesicles, unfilled circles show the $\left[P L A_{1 A}\right]$. Figures $2 B, 2 C$ and $2 D$ show the data obtained in 0.5, 1 and 2 wt \% Ficoll 400, respectively. [PLA $\left.{ }_{1 A}\right]_{\text {norm }}$ data show the [PLA $A_{1 A}$ ] normalised to the $\left[P L A_{1 A}\right]_{0}$ i.e. $\left[P L A_{1 A}\right]$ at 0 min for that wt \% Ficoll 400 . Initial concentrations were 0.2 to $0.3 \mathrm{mg} / \mathrm{ml} \mathrm{PLA}_{1}$ and 0.15 to $0.2 \mathrm{mM}$ DOPC vesicles. Data are the mean average of three independent repeats \pm standard deviation. Data fits were obtained using a model developed in COPASI in accordance with the kinetic scheme laid out in Scheme 3 as discussed in Section 3.2.

Figure $2 A$ shows that, when vesicles are present in chamber $B$, there is a decrease in the amount of $\left[P L A_{1 A}\right]$ over time. This is best illustrated by the 0 wt \% data (Figure $2 A$ ), where the control study in the absence of vesicles shows no change in $\left[P L A_{1 A}\right]$. Thus we attribute the decrease of $\left[P L A_{1 A}\right]$ to a change in $\left[P L A_{1 B}\right]$ in chamber $B$, initiated by $P L A_{1}$ binding to the vesicles.

The effect of macromolecular crowding on $\mathrm{PLA}_{1}$ binding to DOPC vesicles is shown most clearly in the normalised plots where, as the wt \% of Ficoll 400 increases from $0,0.5,1$ and $2 \mathrm{wt}$ $\%$, the observed equilibrium $\left[\mathrm{PLA}_{1 \mathrm{~A}}\right]_{\text {norm }}$ decreases in the sequence $0.73 \pm 0.01,0.69 \pm 0.02,0.66$ \pm 0.01 and $0.62 \pm 0.01$. This suggests that molecular crowding increases the association of PLA with the vesicles, assuming $\mathrm{PLA}_{1}$ binding is independent of the final vesicle composition.

\subsection{Modelling the equilibrium association kinetics of $P L A_{1}$ binding to DOPC vesicles in a crowded} macromolecular environment

Figure 2 also shows that the time taken for the $\left[\mathrm{PLA}_{1 \mathrm{~A}}\right]$ to equilibrate is approximately $60,90,120$ and 180 minutes for the $0,0.5,1$ and 2 wt \% Ficoll 400 respectively. This is consistent with macromolecular crowding agents reducing the diffusion coefficients of PLA $\mathrm{A}_{1}$ and the vesicles. To model this phenomenon, which corresponds to steps E1 and E2 in Scheme 3, we used the biochemical network simulator COPASI, see Figures S14 and S15 for differential 
equations. This enabled us to fit the data in Figure 2 and determine the equilibrium binding constant $\left(K_{e q}\right)$, equal to $k_{1} / k_{-1}$, using Equation 1.

$$
K_{e q}=\frac{\left[P L A_{1 B} V\right]}{\left[P L A_{1 B}\right][V]_{e q}}=\frac{2\left(\left[P L A_{1}\right]_{0}-\left[P L A_{1 A}\right]\right)}{\left[P L A_{1 A}\right][V]_{e q}}
$$

Equation 1.

Where $\left[P L A_{1 A}\right]_{0}$ is the initial concentration of $P L A_{1}$ in chamber $A$ at time zero, $\left[P L A_{1 A}\right]$ is the concentration of $\mathrm{PLA}_{1}$ in chamber $\mathrm{A}$ at equilibrium and $[\mathrm{V}]_{\mathrm{eq}}$ is the concentration of unoccupied binding sites on the vesicle at equilibrium. This assumes that the concentration of $\mathrm{PLA}_{1}$ in chamber $A$ is equal to the concentration of $\mathrm{PLA}_{1}$ that is unbound to the vesicles in chamber $B$, hence the amount of $\mathrm{PLA}_{1}$ taken up by the vesicles (in a single $1 \mathrm{ml}$ chamber) is $2\left(\left[\mathrm{PLA}_{1}\right]_{0}-\left[\mathrm{PLA} \mathrm{A}_{1 \mathrm{~A}}\right]\right)=\mathrm{PLA}_{1 \mathrm{~B}} \mathrm{~V}$.

The model was parameterised using the starting concentrations of $\mathrm{PLA}_{1}$ in chamber $\mathrm{A}$ and chamber $B$, obtained from Figure 2 (0 mins). The equilibrium [PLA $\left.A_{1 A}\right]$ at each wt \% Ficoll 400 was also obtained from Figure 2. To calculate $\mathrm{K}_{\mathrm{eq}}$, the number of vesicle binding sites [V] and their occupancy at equilibrium needs to be estimated. The occupancy of the binding sites at equilibrium is equal to the number of $\mathrm{PLA}_{1}$ molecules bound to a vesicle at equilibrium i.e. $\left[P L A_{1 B} V\right]$ thus $[V]_{e q}=[V]-\left[P L A_{1 B} V\right]$.

[V] can be estimated using the protocols laid down in the surface dilution models developed for $\mathrm{PLA}_{2}$ from the cobra Naja naja naja $[35,36]$, where the upper limit on binding sites is determined by the number of PLA 2 molecules that can physically cover a micelle/ vesicle. We estimated the molar concentration of available binding sites [V] on the vesicle by dividing the cross-sectional area, at the widest point of the crystal structure of PLA from $T$. lanuginosus [59] $\left(\sim 1.7 \times 10^{-17} \mathrm{~m}^{2}\right)$, by the surface area of a DOPC molecule, $76 \AA^{2}$ [25]. Thus one $\mathrm{PLA}_{1}$ (35 kDa) molecule will occupy the space of around 23 DOPC molecules, which compares well with the 7 lipid molecules occupied by the smaller Naja naja naja PLA 2 (11 kDa) [60]. Taking into account the bilayer structure of the vesicles, which halves the available lipid surface 
area, we estimate that for a starting amount of $500 \mu \mathrm{l}$ of $1.5 \mathrm{mM}$ DOPC vesicles (as used to set up the experiment in Figure 2) there are $1.6 \times 10^{-5} \mathrm{M}$ binding sites available. It is worthwhile pointing out that this approach does have the limitation that it effectively treats the system as a single vesicle or as a system, where the area occupied by each lipid is independent of vesicle size, vesicle composition and any interfacial differences therein. This approach cannot easily be avoided and we consider the impact of using such a simple model on our findings later in the manuscript.

This method enabled us to estimate that $\mathrm{K}_{\mathrm{eq}}$ for $\mathrm{PLA}_{1}$ in $0 \mathrm{wt} \%$ is $6.5 \times 10^{4} \mathrm{M}^{-1}$, this is in broad agreement with the calculated dissociation constants for Naja naja naja PLA 2 with micelles $7 \times 10^{-4} \mathrm{M}[36]\left(\mathrm{K}_{\text {eq }}=1.4 \times 10^{3} \mathrm{M}^{-1}\right)$ or bee venom PLA 2 with DPPC vesicles, $2.4 \times 10^{-5} \mathrm{M}$ $\left(K_{\text {eq }}=4.2 \times 10^{4} \mathrm{M}^{-1}\right)$, estimated from Berg et al. [38], for 3500 binding sites. Figure S16 shows the variance of $K_{\text {eq }}$ with wt \% Ficoll 400 , the data are well fit $\left(R^{2}=0.97\right)$ with a linear trend line of gradient $3.1 \times 10^{4}$ and are consistent with the excluded volume increasing the equilibrium association constant [61]. Table 1 shows $\mathrm{K}_{\text {eq }}$ calculated for the different wt \% of crowding agent.

Next we focused on obtaining a good experimental fit for the 0 wt $\%$ data in Figure $2 \mathrm{~A}$, by adjusting the values of $k_{a}, k_{b}, k_{1}$ and $k_{-1}$ but maintaining $K_{D}$ and $K_{\text {eq }}$ constant. It should be noted that at equilibrium the concentration of $\mathrm{PLA}_{1}$ is equal in chamber $\mathrm{A}$ and $\mathrm{B}$ hence the equilibrium constant for transfer between $A$ and $B\left(K_{D}\right)$ is 1 . In general, good fits for the timescale of $\mathrm{PLA}_{1}$ passage through the dialysis membrane were achieved with $\mathrm{k}_{\mathrm{a}}=\mathrm{k}_{\mathrm{b}}=4.0 \times 10^{-5}$ $\min ^{-1}$. However, the slightly sigmoidal shape of the plots could only be fitted when $\mathrm{k}_{1}$ was of the order of $1 \times 10^{3}\left(1 \mathrm{~min}^{-1}\right)$. The final fit parameters are shown in Table 1. Previous work has shown that the association rate constant of PLA $\mathrm{A}_{2}$ with vesicles in uncrowded media is around $0.24 \times 10^{3} \mathrm{~min}^{-1}$ [38], which is in broad agreement with the value of $\mathrm{k}_{1}$ we found to give the best fit.

To model the effect of macromolecular crowding on the association kinetics of PLA 1 with DOPC vesicles we applied a single correction factor (for each wt \% Ficoll 400) to the rate constants $k_{a}, k_{b}, k_{1}, k_{-1}$ obtained at 0 wt \%. We took this approach, which assumes the collision 
of PLA $\mathrm{P}_{1}$ and vesicles is a diffusion controlled process, because the diffusion coefficients of a range of molecules in Ficoll 70 decrease linearly with increasing wt \% Ficoll 70 [6]. The final values used for the fits in Figure 2 are shown in Table 1.

Table 1 Equilibrium association parameters used to fit the data in Figure 2.

\begin{tabular}{|c|c|c|c|c|c|c|}
\hline $\begin{array}{c}\text { wt \% Ficoll } \\
400\end{array}$ & $\mathrm{k}_{\mathrm{a}}\left(\mathrm{min}^{-1}\right)$ & $\mathrm{k}_{\mathrm{b}}\left(\mathrm{min}^{-1}\right)$ & $\mathrm{K}_{\mathrm{D}}$ & $\mathrm{k}_{1}\left(\mathrm{Imol} \mathrm{min}^{-1}\right)$ & $\mathrm{k}_{-1}\left(\mathrm{~min}^{-1}\right)$ & $\mathrm{K}_{\text {eq }}\left(\mathrm{M}^{-1}\right)$ \\
\hline 0 & $4.0 \times 10^{-5}$ & $4.00 \times 10^{-5}$ & 1 & $2.48 \times 10^{3}$ & 0.038 & $6.5 \times 10^{4}$ \\
\hline 0.5 & $3.4 \times 10^{-5}$ & $3.4 \times 10^{-5}$ & 1 & $2.44 \times 10^{3}$ & 0.028 & $8.8 \times 10^{4}$ \\
\hline 1 & $3.2 \times 10^{-5}$ & $3.2 \times 10^{-5}$ & 1 & $2.24 \times 10^{3}$ & 0.024 & $9.2 \times 10^{4}$ \\
\hline 2 & $2.4 \times 10^{-5}$ & $2.4 \times 10^{-5}$ & 1 & $1.73 \times 10^{3}$ & 0.014 & $1.3 \times 10^{5}$ \\
\hline
\end{tabular}

Previous work in Ficoll 400 has shown that at 6.9 wt \% the diffusion coefficient of fluorescent tagged Ficoll molecules (tracer molecules) drops by a factor of 0.42 , when compared to the diffusion coefficient in 0 wt \% Ficoll 400. The authors note a weak size dependence [62], which may explain why in our model a factor of 0.6 applied to $k_{a}, k_{b}, k_{1}$ and $k_{-1}$ gives a good fit for the $2 \mathrm{wt} \%$ crowded solution in Figure 2D.

3.3 Macromolecular crowding decreases the initial reaction velocity of PLA $A_{1}$ and decreases the apparent reaction equilibrium

Using a mass spectrometric assay, we assessed the activity of PLA ${ }_{1}$ towards DOPC vesicles at different concentrations of crowding agent and in the absence of crowding agent. These assays were set up to have similar starting $\mathrm{PLA}_{1}$ and DOPC concentrations to those in chamber B of the equilibrium dialysis experiments. To a first order approximation, this approach makes the results of the two experiments directly comparable. Figure 3 shows the results of these experiments. 

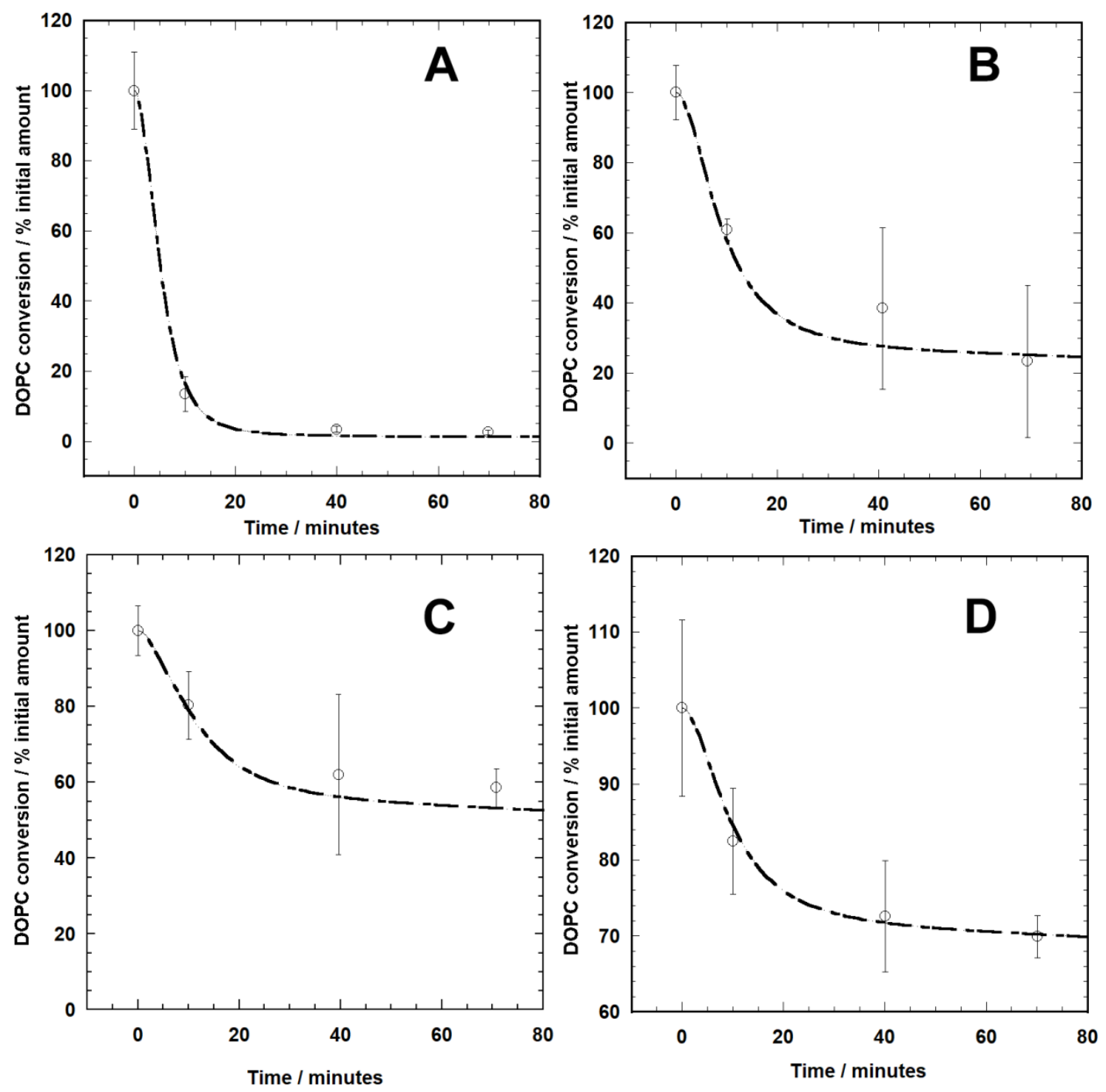

Figure 3 the time-dependent activity of $\mathrm{PLA}_{1}$ against DOPC lipid vesicles in a macromolecular crowded environment. Figures $3 A, 3 B, 3 C$ and 3D show data obtained in $0,0.5,1$ and 2 wt \% Ficoll 400 respectively. Data show the percentage of DOPC remaining in the reaction mixtures with respect to time. Initial PLA 1 and DOPC concentrations were $0.35 \mathrm{mg} / \mathrm{ml}$ and $0.2 \mathrm{mM}$, respectively. All studies were performed in triplicate, error bars show the standard deviation of the mean average conversion. Data were fit using the general model developed later in the manuscript. 
In the absence of crowding agent, Figure 3 shows that 95\% of the initial DOPC has been converted to OA and OPC within 20 minutes. The high level of conversion suggests that under the conditions of the study the vesicles undergo significant fusion and redistribution, since only half the available substrate (i.e. the lipid in the outer bilayer membrane leaflet) is available at the start of the reaction [63]. The initial rate of reaction is $1.7 \times 10^{-8} \mathrm{moles} \mathrm{min}^{-1}$ in $0 \mathrm{wt} \%$ Ficoll, when calculated for the first 10 minutes of the reaction. Estimates of the initial rates at $0.5,1$

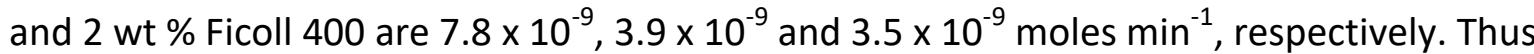
Figure 3 demonstrates that as the wt \% of Ficoll 400 increases the rate of reaction decreases. This result is a little surprising since we have also shown that macromolecular crowding increases the binding of $\mathrm{PLA}_{1}$ to the vesicles, thus we anticipated an increase in the rate of reaction.

Another interesting feature of the data is that as macromolecular crowding increases, premature cessation of the reaction occurs, since the apparent reaction equilibrium reduces from $95 \%$ to $70 \%$ to $55 \%$ and $30 \%$ DOPC conversion at $0,0.5,1$ and 2 wt $\%$ Ficoll 400 , respectively. This reduction in the consumption of DOPC is not easily explained by a combination of changing vesicle dynamics and the reduced diffusion coefficients of PLA $\mathrm{A}_{1}$ and vesicles in Ficoll 400, since at least half the initial DOPC will be always accessible to PLA . $_{\text {. }}$ Furthermore, the decrease in conversion of DOPC to OPC and OA correlates inversely with increasing $\mathrm{PLA}_{1}$ binding in Figure 2 , suggesting that the increased binding of $\mathrm{PLA}_{1}$ is not due to product formation (or an associated property of the changing membrane interface).

Interestingly, the kinetics of product formation do correlate with the time taken to reach equilibrium binding in the dialysis experiment in Figure 2 , such that the fastest reactions equilibrate most quickly. This could indicate that the reaction products (OA and OPC) accelerate binding of $\mathrm{PLA}_{1}$ to the membrane surface. To test this we determined the initial rate of PLA $1 \mathrm{~A}$ decrease in Figure 2. This was $-0.0013,-0.0011,-0.0013$ and $-0.0012 \mathrm{mgml}^{-1} \mathrm{~min}^{-1}$ at $0,0.5,1$ and 2 wt\% Ficoll 400, respectively. These figures do not correlate with the initial rates of OA production, suggesting the reaction products do not accelerate binding of PLA 1 to the vesicle surfaces. On this topic, it is worth noting that the equilibrium dialysis studies were set up with 
$\mathrm{PLA}_{1}$ equilibrated between both chamber $A$ and $B$, prior to the reaction initiation with DOPC vesicles. Whilst, the enzyme assay of PLA 1 activity was carried out under very similar experimental conditions to those in chamber B of the equilibrium dialysis experiments in Figure 2. This means that the timescales of the hydrolysis of DOPC in the two systems are comparable. These are not ideal experimental conditions to explore whether or not the reaction products accelerate binding of $\mathrm{PLA}_{1}$, since $\mathrm{PLA}_{1 \mathrm{~B}}$ likely binds to the vesicles more rapidly than $\mathrm{PLA} \mathrm{A}_{1 \mathrm{~A}}$ diffuses through the dialysis membrane, (a process that takes several hours).

To investigate the possibility that $\mathrm{PLA}_{1}$ undergoes significant structural changes due to macromolecular crowding, which might explain the trends seen in Figure 3, we carried out a differential scanning calorimetry study of $\mathrm{PLA}_{1}$ in the presence of different weight percentages of Ficoll 400 . These results showed that the $T_{m}$ of $\mathrm{PLA}_{1}\left(\operatorname{circa} 70^{\circ} \mathrm{C}\right)$ is unchanged by up to 8 wt\% Ficoll 400, as shown in Figure S17, suggesting structural changes to PLA $A_{1}$ cannot account for the change in $\mathrm{PLA}_{1}$ activity in the presence of crowding agents.

The trends in Figure 3 are very similar to those observed for human cytosolic phospholipase $A_{2}\left(c P L A_{2}\right)$ [63], where the increasing radius of vesicles correlates with a reduction in the apparent equilibrium constant. The authors [63] suggest that this occurs because the active membrane bound form of $\mathrm{CPLA}_{2}$ is deactivated by either the radius or the curvature of the vesicle. Similar membrane curvature dependent behaviour has been observed for $\alpha$-synuclein [64], acyl-coA binding protein [65] and tocopherol transfer protein [66] binding to membranes. We, therefore, investigated the effect of Ficoll 400 on the size distribution of vesicles in the presence and absence of PLA $\mathrm{PL}_{1}$ to ascertain if any of the effects of vesicle size, seen for PLA $2[63,67]$, might impact the activity of PLA $A_{1}$ in our system.

\subsection{Evidence that macromolecular crowding drives vesicle aggregation in the presence of PLA 1}

Dynamic light scattering (DLS) experiments were set up to broadly replicate the experimental conditions of the mass spectrometry studies shown in Figure 3. Figure 4 shows 
the effect of PLA $A_{1}$ action on DOPC vesicles, compared to control studies where no PLA is $_{1}$ present.
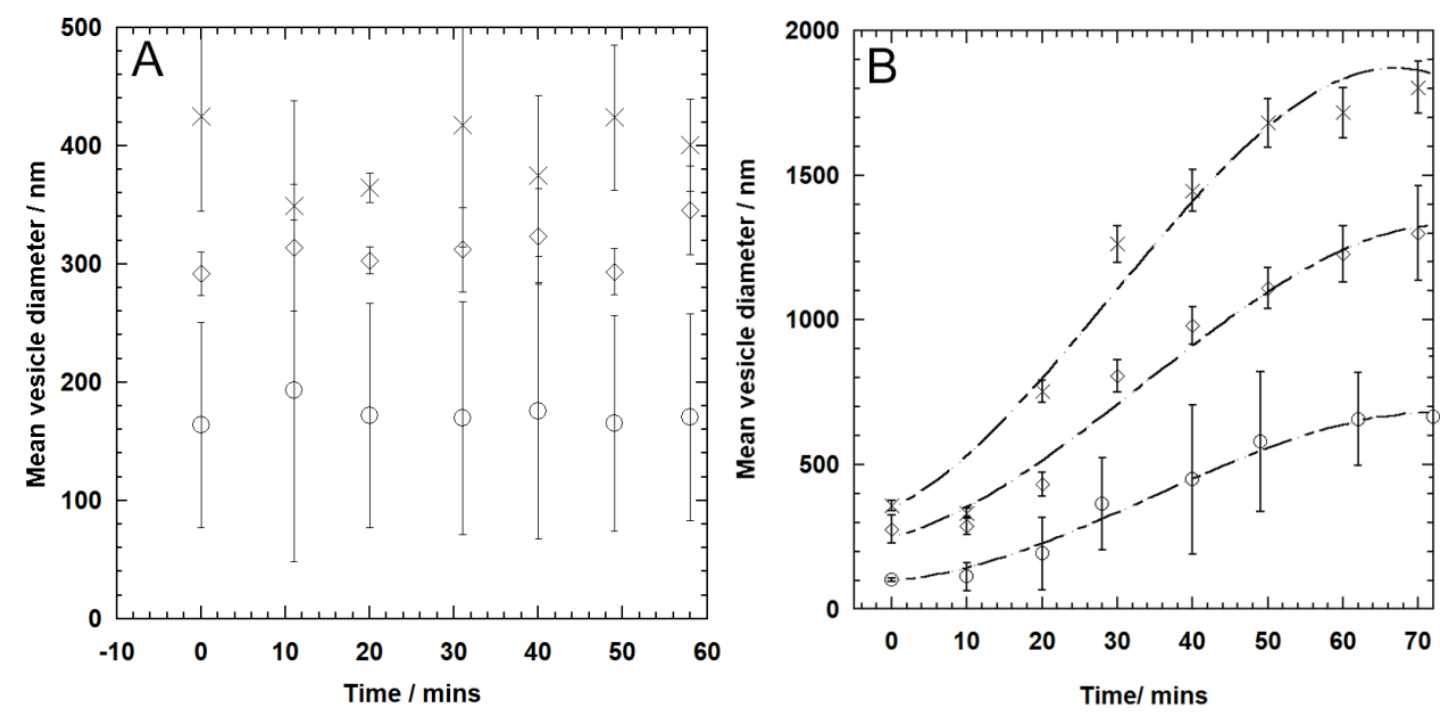

Figure 4 the action of $\mathrm{PLA}_{1}$ on DOPC vesicles studied by dynamic light scattering. Initial PLA $\mathrm{A}_{1}$ and DOPC concentrations were $0.2 \mathrm{mg} / \mathrm{ml}$ and $0.15 \mathrm{mM}$, respectively. Plots show the mean average vesicle diameter versus time. Three independent repeats were performed and error bars are \pm standard deviation. Figure $4 \mathrm{~A}$ shows control studies in the absence of $\mathrm{PLA}_{1}$ at 0 wt \% (empty circles), 1 wt \% (empty diamonds) and 2 wt \% (crosses) Ficoll 400. Figure 4B shows vesicle size changing in the presence of PLA 1 at 0 wt \% (empty circles), 1 wt \% (empty diamonds) and 2 wt $\%$ (crosses) Ficoll 400, respectively. Data series were fit, over the range of the plots, using $3^{\text {rd }}$ order polynomials with the coefficients summarised in Table S1.

Figure $4 \mathrm{~A}$ shows very clearly that in the absence of $\mathrm{PLA}_{1}$, at all wt \% Ficoll 400 , the mean average vesicle size is stable w.r.t. time. However, the vesicles formed have different average sizes of 160, 300 and $400 \mathrm{~nm}$ when prepared in 0,1 and 2 wt \% Ficoll 400, respectively. This suggests that the higher initial velocity of $\mathrm{PLA}_{1}$ at $0 \mathrm{wt} \%$ Ficoll 400 might be because PLA $\mathrm{A}_{1}$ is more active towards smaller vesicles, as is the case with porcine pancreatic and bee venom $\mathrm{PLA}_{2}[67]$. 
Figure 4B shows how the mean vesicle size changes after addition of $\mathrm{PLA}_{1}$. It is clear that as the wt \% of Ficoll 400 increases the apparent vesicle size increases more rapidly w.r.t. time. This is interesting because in Figure 3 we observe an early cessation of the reaction as the wt \% of Ficoll 400 increases. Thus the data suggest the increasing vesicle size might be inhibiting PLA 1 activity, although it should be noted that the size increase observed by DLS could be explained by vesicles aggregating but not fusing into single vesicles. This is particularly relevant given the known effects of calcium on anionic lipids and the changing chemical nature of the bilayer membrane interface during $\mathrm{PLA}_{1}$ action. Figure $4 \mathrm{~A}$ shows that vesicle diameter does not change in the absence of PLA 1 , thus our results show very clearly that PLA 1 plays a role in initiating the apparent vesicle growth. This is consistent with studies that have concluded that vesicle fission and vesicle fusion are critical aspects of PLA $\mathrm{A}_{2}$ kinetics [38,67-69] when anionic lipids and high levels of calcium ions are present. Furthermore, other studies show that the introduction of fatty acids and lysolipids (in the absence of protein) also promotes fusion between vesicles [70].

From the data in Figure 4 we hypothesised that, as the anionic lipid OA is produced by $\mathrm{PLA}_{1}$, the relative rates of vesicle fission and vesicle fusion increase but macromolecular crowding favours increases in the rate of vesicle fusion over the rate vesicle fission, leading to larger vesicles forming. To look for evidence that might support this hypothesis, we determined the size distribution of the vesicles in the presence of macromolecular crowding agents and $\mathrm{PLA}_{1}$. These data, shown in Figure 5, confirmed that the smaller vesicles disappear more quickly, as the wt \% of Ficoll 400 increases when $\mathrm{PLA}_{1}$ is present. The mean average polydispersity index and standard deviation of the initial vesicle preparations were $0.84 \pm 0.06$, $0.95 \pm 0.09$ and $0.85 \pm 0.1$ for 0,1 and 2 wt\% Ficoll 400 , respectively. Final values of the mean average polydispersity index were $0.40 \pm 0.06,0.46 \pm 0.09$ and $0.49 \pm 0.1$ for 0,1 and 2 wt $\%$ Ficoll 400, respectively. Thus all samples show a decrease in polydispersity that is consistent with the loss of the smaller vesicles in the population. 

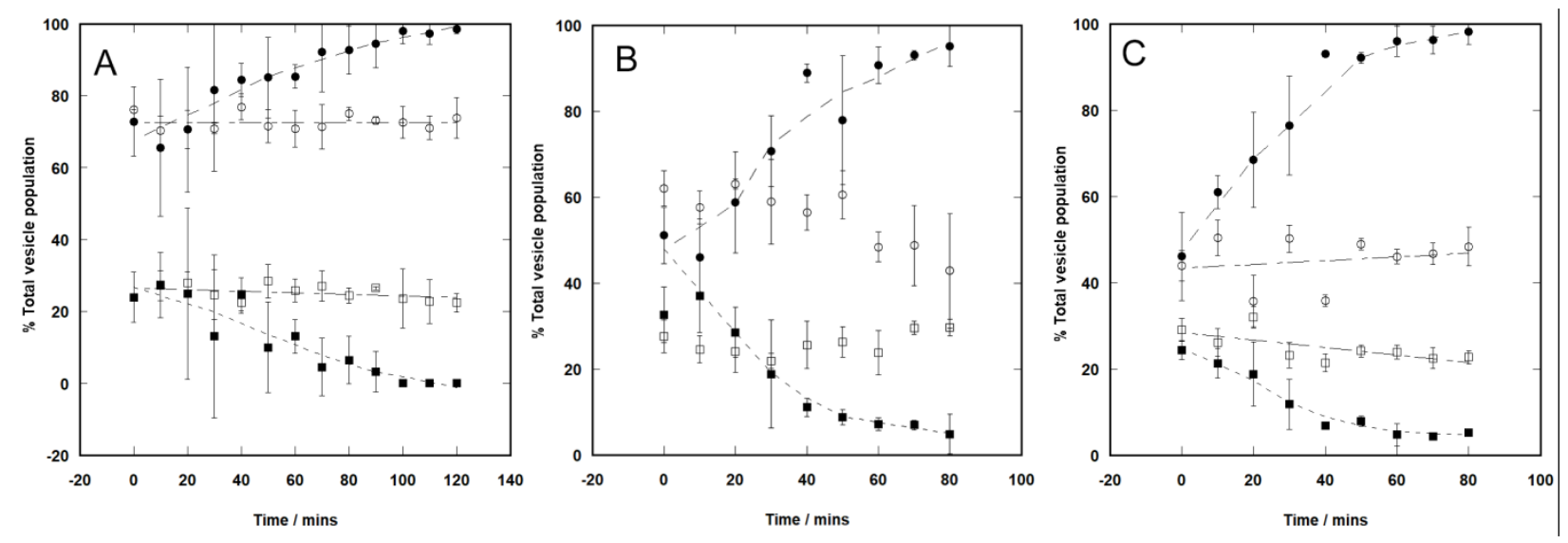

Figure 5 The time-dependent behaviour of different sized populations of vesicles treated with $\mathrm{PLA}_{1}$ at different wt\% of Ficoll 400. Figures 5A, 5B and 5C show 0, 1 and 2 wt\% Ficoll 400, respectively. Circles show vesicles in the size range of 1 to $2 \mu \mathrm{m}$ diameter. Squares show vesicles in the size range 0.2 to $0.4 \mu \mathrm{m}$. Unfilled shapes show data for vesicles in the absence of $\mathrm{PLA}_{1}$ and filled shapes show data in the presence of PLA $A_{1}$. Data show that the action of PLA with increasing wt\% of Ficoll 400, decreases the number of smaller vesicles in solution more rapidly. Data points are the average of three independent repeats plus/ minus the standard deviation.

The data in Figure 5 are consistent with the excluded volume effect favouring the aggregation of vesicles, which has been observed previously [71]. The data also suggest

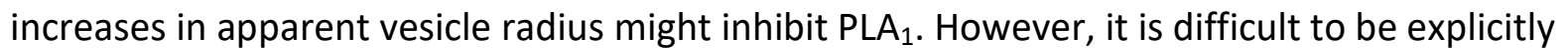
sure of this since it might be the case that the larger vesicles are still substrates for PLA but are $^{2}$ less prone to fusion. To address these observations in a more quantitative, way we extended our equilibrium model in COPASI to see if we might be able to fit the $\mathrm{PLA}_{1}$ activity data in Figure 3 by incorporating a term relating to apparent vesicle size observed by DLS.

\section{5 $\mathrm{A}$ model for the modulation of $P L A_{1}$ activity by vesicle radius in the presence of} macromolecular crowding agents

Using COPASI we set up a single compartment model for Scheme 3, see Figure S15 for differential equations. The first step E1 where PLA $A_{1}$ crosses the dialysis membrane was omitted, 
in keeping with the experimental set up for the mass spectrometry assay. Step E2 was parameterised using the equilibrium association and rate constants in Table 1 for each wt \% Ficoll 400. To model vesicle size dependent modulation of $\mathrm{PLA}_{1}$, we introduced the parameter $(\gamma)$, defined in Equation 2.

$$
\gamma=\left(\frac{d_{0}}{d_{t}}\right)
$$

Equation 2,

Where $d_{0}$ is the average vesicle diameter (in $\mathrm{nm}$ ) at $\mathrm{t}=0$ in the $0 \mathrm{wt} \%$ Ficoll 400 buffer, equal to $100 \mathrm{~nm}$ and $d_{t}$ is the average vesicle diameter at time $t$. This approach establishes $\gamma$ as a parameter in the model so that the rate constants in the reaction vary with apparent vesicle diameter. The forward rate constants $\left(k_{2}\right.$ and $k_{3}$ ) vary proportionally to $\gamma$ and the reverse rate constant $\left(k_{-2}\right)$ varies as $1 / \gamma$. The rate constant $k_{-3}$ was kept constant since it is very small and thus changes to it have negligible effect.

We found that using the variance of $d_{t}$ with time, parameterised from the cubic fits of the sizing data shown in Table S1, a general model could be applied, such that;

$$
d_{t}=a t^{3}+b t^{2}+c t+d \quad \text { Equation } 3
$$

Where $t$ is the time in minutes and $a, b, c$ and $d$ are linear functions of the wt \% Ficoll $400(x)$, with values of these constants shown in Table 2. In this model $k_{-2}$ decreases linearly with wt \% Ficoll 400. 
Table 2 rate constants used in the data fitting for Figure 3 . Values of $a, b, c, d$ and $k_{-2}$ are from a general linear equation of the form $y=m x+c$, where $x$ is the wt \% Ficoll 400 present in the buffer.

\begin{tabular}{|c|c|c|c|c|c|}
\hline & $m$ & C & $\mathrm{k}_{2}\left(\mathrm{I} \mathrm{mol}^{-1} \mathrm{~min}^{-1}\right)$ & $k_{3}\left(\min ^{-1}\right)$ & $\mathrm{k}_{-3}\left(\mathrm{I}^{2} \mathrm{~mol}^{-2} \mathrm{~min}^{-2}\right)$ \\
\hline $\mathbf{a}$ & $-2.3 \times 10^{-3}$ & $-2.4 \times 10^{-3}$ & $1.1 \times 10^{6}$ & $7.1 \times 10^{5}$ & 9.96 \\
\hline b & 0.18 & 0.26 & & & \\
\hline c & 5.35 & 1.72 & & & \\
\hline d & 130 & 100 & & & \\
\hline$k_{-2}$ & $-1.95 \times 10^{5}$ & $5.7 \times 10^{5}$ & & & \\
\hline
\end{tabular}

This general model enabled us to fit the trends we see in $\mathrm{PLA}_{1}$ activity in Figure 3 , which were consistent with our working hypothesis that larger vesicles, which form more quickly in

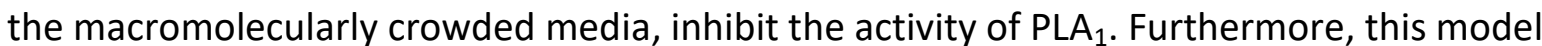
allows us to understand the likely origin of some of the error observed in Figure 3, which is probably down to the sensitivity of PLA 1 activity to the size distribution of the vesicles over the course of the reaction.

To arrive at this conclusion we have made a number of assumptions about the nature and number of binding interactions between PLA $\mathrm{A}_{1}$ and DOPC vesicles. We now consider the effect of these assumptions on our findings, although it is worth pointing out that inaccuracies in the estimation of the number of binding sites cannot account for the change in apparent reaction equilibrium observed under different amounts of macromolecular crowding, Figure 3. This is because the number of binding sites determines the time taken for the reaction to reach equilibrium and not apparent position of the chemical equilibrium. 


\subsection{Evaluating assumptions in the COPASI model}

In our estimates we have assumed that both the total number and occupancy of the binding sites does not change across the reaction coordinate. This approach is consistent with published surface dilution models $[35,36,60]$. However, it is worthwhile considering the consequences of these assumptions on the analysis presented.

\subsubsection{Assumptions relating to the absolute number of binding sites [V]}

By assuming [V] is constant we effectively assume that the total available vesicle surface area is independent of the size of the vesicles, their chemical composition and any associated interfacial effects, for a fixed starting lipid amount. This necessity of this approach can be demonstrated by a simple calculation of the total available surface area of the vesicles, from the molecular cross-sectional area of the lipids. For a fixed starting lipid amount of $1 \times 10^{-3}$ moles, there are $6.02 \times 10^{20}$ molecules of DOPC, which corresponds to a surface area of $4.60 \mathrm{~m}^{2}$ based on a DOPC molecular cross-sectional area of $76 \times 10^{-20} \mathrm{~m}^{2}$ [25]. This total surface could be divided across two vesicles of two different radii, say $20 \times 10^{-9} \mathrm{~m}$ and $200 \times 10^{-9} \mathrm{~m}$, which have surface areas (calculated as $4 \pi \mathrm{r}^{2}$ ) of $5.0 \times 10^{-15} \mathrm{~m}^{2}$ and $5.0 \times 10^{-13} \mathrm{~m}^{2}$ to make $9.2 \times 10^{14}$ and $9.2 \mathrm{x}$ $10^{12}$ vesicles, respectively, assuming an equal distribution of lipids between the two membrane leaflets. As the reactions progresses the area occupied per lipid headgroup, for both reactants and products, might change with composition and vesicle radius. However, without this information, the total available surface area of the lipids in the system cannot be calculated and the number of vesicles and PLA 1 binding sites cannot be determined more accurately.

As a result several key geometric properties of vesicles are not accounted for by this approach, which in turn leads to inaccuracies in the quantification of PLA $A_{1}$ binding sites. For example, a geometric analysis shows that for small vesicles of circa $30 \mathrm{~nm}$ diameter there are roughly a factor of 1.4 more DOPC lipids on the outer than inner membrane leaflet [72], for a bilayer of $4.6 \mathrm{~nm}$ thickness. As the vesicle radius increases the ratio of inner to outer leaflet lipids tends to $1: 1$. Thus the total surface area of lipid available, and hence the number of 
binding sites, is weakly dependent on vesicle size for a fixed amount of lipid. In our study we estimate a ratio of inner to outer leaflet lipid of 1.05 for $200 \mathrm{~nm}$ and 1.02 for $500 \mathrm{~nm}$ vesicles. Incorporating these small changes has no effect on the results we have presented.

Whilst there is evidence to suggest that above a radius of $110 \AA$ lipid molecules occupy the same cross-sectional area in PC vesicles [73], our assumption that lipid molecules occupy the same cross-sectional area in vesicles regardless of the vesicle radius is also important to consider due to the geometric properties of fusing vesicles. This necessitates further assumptions to be made about the number of available PLA 1 binding sites. Since when two vesicles fuse, assuming the volume of their contents is not lost; the total surface area of the new fused vesicle is less than that of the initial vesicles. In fact it can easily be shown that for two spherical vesicles of equal volume fusing there is a factor of 0.79 reduction in net surface area, Figure S18. For vesicles of different radii fusing this factor increases and the associated decrease in binding sites is less significant. In principle this decrease in vesicle surface area can cause a decrease in the number of PLA $\mathrm{P}_{1}$ binding sites [V], either at the start of the reaction for vesicles of different radii (formed by fusion) or during the reaction if there are fusion events occurring. Therefore, we carried out an analysis to ascertain if the variance in binding sites could account for any of the conclusions in this manuscript.

Assuming no lipids are lost and local spherical curvature is preserved in the final fused vesicles the decrease in total vesicle surface area requires a decrease in the surface area occupied per lipid to occur. Hence the lower limit of PLA ${ }_{1}$ binding sites is related to the minimum area per lipid headgroup that can be occupied before either fusion does not occur, local areas of non-spherical curvature occur, or lipids are lost from the vesicle. Studies show that PC lipids do not form bilayer vesicles with radii less than $80 \AA$, where the area per lipid is circa $36 \AA$. To a first order approximation this suggests that the minimal surface area of fusing vesicles in our study is proportional to the maximum compression factor experienced by the headgroups i.e. $76 / 36$ or roughly a factor of two, where $76 \AA$ is a typical area occupied by a DOPC lipid in a vesicle [25]. This means that as the reaction progresses the number of binding sites could decrease by a maximum factor of two through the reaction as a result of vesicle 
fusion. Halving the number of binding sites and rerunning the simulations gives rise to very similar data fits. Running the 0 wt\% Ficoll 400 simulation with half the number of binding sites does not give rise to good 'fits' for the $0.5,1$ or 2 wt\% Ficoll 400 data suggesting that our findings are not impacted by keeping [V] constant.

Similarly, the concentration of binding sites for $\mathrm{PLA}_{1}$ is related to half the available vesicle surface. This effectively assumes that during the normal dynamics of vesicle fusion and lipid exchange PLA $\mathrm{P}_{1}$ is not internalised in any vesicles. This is consistent with previous models; however we tested the validity of this assumption by doubling [V], which had no impact on the conclusions presented. Changes to the magnitude of the rate constants $\left(k_{1}, k_{-1}, k_{2}, k_{-2}, k_{3}\right.$ and $k_{-}$ 3) were required.

\subsubsection{Assumptions relating to the occupancy of binding sites}

Our models also require a number of assumptions about the occupancy of [V] to be made. For example, we have assumed there are no significant interactions whereby Ficoll 400 molecules might displace PLA 1 bound to a vesicle. The effects of Ficoll 400 on biological membranes are not well studied, however this appears to be supported by our findings in Figure 3, where we see a clear indication of an increase in $\mathrm{PLA}_{1}$ binding to DOPC vesicles with increasing wt \% Ficoll 400. Whereas, if Ficoll 400 competitively displaced PLA $_{1}$ it is anticipated that as the wt \% Ficoll 400 increases the amount of PLA 1 bound would decrease.

We have also assumed that both the number and occupancy of binding sites [V] is independent of the chemical composition of the vesicles. However it is possible that as the reaction progresses, compositional changes cause the surface area and biophysical properties of the vesicles to vary. For example, OA carries a negative charge and there is some evidence that negative lipids increase the binding of phospholipases to membranes [39]. Thus as the reaction proceeds and more $\mathrm{OA}$ is made, $\mathrm{PLA}_{1}$ binding to the vesicles will increase. Similarly, theory predicts stored elastic energy in the bilayers of the vesicles will increase as DOPC is converted to OA and OPC, which is essentially driven by the negative spontaneous curvature 
preference of OA in DOPC lipid systems [29,31], see Figure S19 and Section S6.1 for an estimate. However, since both charge and stored elastic energy increase with reaction progress and we observe an increase in $\mathrm{PLA}_{1}$ binding that correlates with reduced reaction rate, it would appear that our assumption is reasonable.

\section{Conclusions}

We have shown that, consistent with theory, $[74,75]$ macromolecular crowding increases the equilibrium association constant of PLA $\mathrm{A}_{1}$ with vesicles and decreases the association/ dissociation rate constants. This is consistent with the excluded volume effect favouring the aggregated state and the known effects of macromolecular crowding agents on the diffusion coefficients of other molecules. Contrary to expectation, we have shown that the initial activity of PLA 1 towards DOPC vesicles is decreased by macromolecular crowding and an early cessation of the reaction occurs. This was not anticipated since the increased amount of $\mathrm{PLA}_{1}$ bound to the vesicles ought to increase activity through to the same reaction equilibrium.

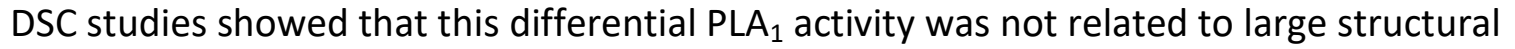
changes in the proteins caused by macromolecular crowding. DLS showed that, as PLA 1 reacts with vesicles, increased macromolecular crowding favours the formation of vesicles with larger radii more rapidly. With this information we developed a more complex kinetic equilibrium model to fit the PLA ${ }_{1}$ activity data, which was consistent with all of our data.

What is less clear is which biophysical property of the vesicles PLA $A_{1}$ is sensing and how this affects the rate constants for steps E3 and E4 in Scheme 3. In broad terms it is possible that the observed drop in $\mathrm{PLA}_{1}$ activity with increasing vesicle diameter is analogous to the relationship between lipid surface pressures in the Langmuir trough and PLA ${ }_{1}$ activity [55]. And it is easy to suggest that the surface pressure of the lipids closes the 'lid' motif on the lipase, deactivating it. This would be consistent with the results we present in this study; however there are currently no methods to determine the surface pressure in lipid vesicles, which makes 
this comparison difficult to quantify. The surface area occupied by lipid headgroups plays an important role in the mechanosensing properties of $\mathrm{PLA}_{2}$ and its activity in osmotically stressed vesicles [27]. Likewise the Gaussian curvature of vesicles impacts the stored elastic energy of membranes, which suggests that this biophysical property may play a role in modulating PLA activity. However, the changing chemical composition of the bilayer interface due to PLA 1 action and the possibility that the products may form laterally segregated domains, introduces another level of complexity into the system studied. For example, there is possibility that in the crowded state, membrane defects and/or product clustering in the membrane, driven by the high calcium used in this study complexing with the OA produced, slow the kinetics of product release by the enzyme ( $k_{3}$, Scheme 3$)$. Or alternatively the release of the enzyme into solution is slowed. The complex nature of this relationship warrants its own detailed study, which will have to be the subject of further work

One of the interesting aspects of this study is that it suggests that the effects of macromolecular crowding agents on vesicle systems, particularly the dynamics of vesicle aggregation, changes in Gaussian curvature and associated biophysical parameters, are an important area for future research. Given the importance of phospholipases in the generation of intracellular vesicles and the role of these in neurocognitive processes and cellular transport it may be that a more detailed understanding of the aggregation dynamics of lipid assemblies under conditions of macromolecular crowding can provide new insights into this biological phenomenon and any associated health disorders.

\section{Acknowledgements}

The authors would like to thanks Tom Hamilton for assistance in setting up DSC studies and Lisa O'Rourke for proof reading the manuscript. 


\section{References}

1. Ellis, R. J. 2001 Macromolecular crowding: obvious but underappreciated. Trends Biochem. Sci. 26, 597-604.

2. Minton, A. P. 2014 The effect of time-dependent macromolecular crowding on the kinetics of protein aggregation: a simple model for the onset of age-related neurodegenerative disease. Front. Phys. 2, 48. (doi:10.3389/fphy.2014.00048)

3. Ross, B. M., Moszczynska, A., Erlich, J. \& Kish, S. J. 1998 Phospholipid-metabolizing enzymes in Alzheimer's disease: increased lysophospholipid acyltransferase activity and decreased phospholipase A2 activity. J. Neurochem. 70, 786-793. (doi:10.1046/j.14714159.1998.70020786.x)

4. Sanchez-Mejia, R. O. \& Mucke, L. 2010 Phospholipase A2 and arachidonic acid in Alzheimer's disease. Biochim. Biophys. Acta 1801, 784-790.

(doi:10.1016/j.bbalip.2010.05.013)

5. Verkman, A. S. 2002 Solute and macromolecule diffusion in cellular aqueous compartments. Trends Biochem. Sci. 27, 27-33. (doi:10.1016/S0968-0004(01)02003-5)

6. Dauty, E. \& Verkman, A. S. 2004 Molecular crowding reduces to a similar extent the diffusion of small solutes and macromolecules: Measurement by fluorescence correlation spectroscopy. J. Mol. Recognit. 17, 441-447. (doi:10.1002/jmr.709)

7. Zimmerman, S. B. \& Harrison, B. 1987 Macromolecular crowding increases binding of DNA polymerase to DNA: An adaptive effect. Biochemistry 84, 1871-1875.

(doi:10.1073/pnas.84.7.1871)

8. Minton, A. P. 1999 Adsorption of globular proteins on locally planar surfaces. II. Models for the effect of multiple adsorbate conformations on adsorption equilibria and kinetics. Biophys. J. 76, 176-187. (doi:10.1016/S0006-3495(99)77187-0)

9. Engelke, H., Dorn, I. \& Rädler, J. O. 2009 Diffusion and molecular binding in crowded 
vesicle solutions measured by fluorescence correlation spectroscopy. Soft Matter 5, 4283-4289. (doi:10.1039/b910539e)

10. Hou, S., Trochimczyk, P., Sun, L., Wisniewska, A., Kalwarczyk, T., Zhang, X., WielgusKutrowska, B., Bzowska, A. \& Holyst, R. 2016 How can macromolecular crowding inhibit biological reactions? The enhanced formation of DNA nanoparticles. Sci. Rep. 6, 22033. (doi:10.1038/srep22033)

11. Dong, H., Qin, S. \& Zhou, H.-X. 2010 Effects of macromolecular crowding on protein conformational changes. PLoS Comput. Biol. 6, e1000833. (doi:10.1371/journal.pcbi.1000833)

12. Eggers, D. K. \& Valentine, J. S. 2001 Crowding and hydration effects on protein conformation: a study with sol-gel encapsulated proteins. J. Mol. Biol. 314, 911-922. (doi:10.1006/jmbi.2001.5166)

13. Politou, A. \& Temussi, P. A. 2015 Revisiting a dogma: The effect of volume exclusion in molecular crowding. Curr. Opin. Struct. Biol. 30, 1-6. (doi:10.1016/j.sbi.2014.10.005)

14. Richmond, G. S. \& Smith, T. K. 2011 Phospholipases A 1. Int. J. Mol. Sci. 12, 588-612. (doi:10.3390/ijms12010588)

15. Inoue, A. \& Aoki, J. 2006 Phospholipase A 1 : structure, distribution and function. Future Lipidol. 1, 687-700. (doi:10.2217/17460875.1.6.687)

16. Mishra, M. K., Kumaraguru, T., Sheelu, G. \& Fadnavis, N. W. 2009 Lipase activity of Lecitase ${ }^{\circledR}$ Ultra: characterization and applications in enantioselective reactions. Tetrahedron: Asymmetry 20, 2854-2860. (doi:10.1016/j.tetasy.2009.11.012)

17. Wang, F., Zhang, H., Liu, Y., Khan, F. I., Yang, B. \& Wang, Y. 2016 Profiling substrate specificity of Lecitase Ultra to different kinds of phospholipids using monolayer technology. Eur. J. Lipid Sci. Technol. , 1-5. (doi:10.1002/ejlt.201600175)

18. Baeza-Jiménez, R., López-Martínez, L. X., Otero, C., Kim, I. H. \& García, H. S. 2013 
Enzyme-catalysed hydrolysis of phosphatidylcholine for the production of lysophosphatidylcholine. J. Chem. Technol. Biotechnol. 88, 1859-1863. (doi:10.1002/jctb.4040)

19. Kim, I. H., Garcia, H. S. \& Hill, C. G. 2007 Phospholipase A1-catalyzed synthesis of phospholipids enriched in $\mathrm{n}-3$ polyunsaturated fatty acid residues. Enzyme Microb. Technol. 40, 1130-1135. (doi:10.1016/j.enzmictec.2006.08.018)

20. Borné, J., Nylander, T. \& Khan, A. 2002 Effect of lipase on different lipid liquid crystalline phases formed by oleic acid based acylglycerols in aqueous systems. Langmuir 18, 89728981. (doi:10.1021/la020377d)

21. Misiūnas, A., Talaikytė, Z., Niaura, G., Razumas, V. \& Nylander, T. 2008 Thermomyces lanuginosus lipase in the liquid-crystalline phases of aqueous phytantriol: X-ray diffraction and vibrational spectroscopic studies. Biophys. Chem. 134, 144-156. (doi:10.1016/j.bpc.2008.02.002)

22. Fagone, P., Sriburi, R., Ward-Chapman, C., Frank, M., Wang, J., Gunter, C., Brewer, J. W. \& Jackowski, S. 2007 Phospholipid biosynthesis program underlying membrane expansion during B-lymphocyte differentiation. J. Biol. Chem. 282, 7591-7605.

(doi:10.1074/jbc.M608175200)

23. Epand, R., Hughes, D., Sayer, B., Borochov, N., Bach, D. \& Wachtel, E. 2003 Novel properties of cholesterol-dioleoylphosphatidylcholine mixtures. Biochim. Biophys. ActaBiomembr. 1616, 196-208. (doi:10.1016/j.bbamem.2003.08.006)

24. Helfrich, W. 1973 Elastic properties of lipid bilayers: theory and possible experiments. $Z$. Naturforsch. C. 28, 693-703. (doi:10.1002/mus.880040211)

25. Attard, G. S., Templer, R. H., Smith, W. S., Hunt, A. N. \& Jackowski, S. 2000 Modulation of CTP:phosphocholine cytidylyltransferase by membrane curvature elastic stress. Proc. Natl. Acad. Sci. U. S. A. 97, 9032-9036. (doi:10.1073/pnas.160260697)

26. Ces, O. \& Mulet, X. 2006 Physical coupling between lipids and proteins: a paradigm for 
cellular control. Signal Transduct. 6, 112-132. (doi:10.1002/sita.200500079)

27. Lehtonen, J. Y. A. \& Kinnunen, P. K. J. 1995 Phospholipase A2 as a mechanosensor. Biophys. J. 68, 1888-1894. (doi:10.1016/S0006-3495(95)80366-8)

28. Brzozowski, A. M., Savage, H., Verma, C. S., Turkenburg, J. P., Lawson, D. M., Svendsen, A. \& Patkar, S. 2000 Structural origins of the interfacial activation in Thermomyces (Humicola) lanuginosa lipase. Biochemistry 39, 15071-15082. (doi:10.1021/bi0013905)

29. Gillams, R. J., Nylander, T., Plivelic, T. S., Dymond, M. K. \& Attard, G. S. 2014 Formation of inverse topology lyotropic phases in dioleoylphosphatidylcholine/oleic Acid and dioleoylphosphatidylethanolamine/oleic Acid binary mixtures. Langmuir 30, 3337-44. (doi:10.1021/la404275u)

30. Bergstrand, N. \& Edwards, K. 2001 Aggregate structure in dilute aqueous dispersions of phospholipids, fatty acids, and lysophospholipids. Langmuir 17, 3245-3253. (doi:10.1021/la010020u)

31. Dymond, M. K., Gillams, R. J., Parker, D. J., Burrell, J., Labrador, A., Nylander, T. \& Attard, G. S. 2016 Lipid Spontaneous Curvatures Estimated from Temperature-Dependent Changes in Inverse Hexagonal Phase Lattice Parameters: Effects of Metal Cations. Langmuir 32, 10083-10092. (doi:10.1021/acs.langmuir.6b03098)

32. Graham, I., Gagne, J. \& Silvius, J. R. 1985 Kinetics and Thermodynamics of CalciumInduced Lateral Phase Separations in Phosphatidic Acid Containing Bilayers. Biochemistry 24, 7123-7131. (doi:10.1021/bi00346a016)

33. Silvius, J. R. \& Gagné, J. 1984 Calcium-Induced Fusion and Lateral Phase Separations in Phosphatidylcholine-Phosphatidylserine Vesicles. Correlation by Calorimetric and Fusion Measurements. Biochemistry 23, 3241-3247. (doi:10.1021/bi00309a019)

34. Ortiz, A. \& Gomez-Fernandez, Juan, C. 1988 Calcium-induced aggregation of phosphatidylcholine vesicles containing free oleic acid. Chem. Phys. Lipids 46, 259-266. 
35. Carman, G. M., Deems, R. A. \& Dennis, E. A. 1995 Lipid signaling enzymes and surface dilution kinetics. J. Biol. Chem. 32, 18711-18714.

36. Deems, R. A., Eaton, B. R. \& Dennis, E. A. 1975 Kinetic analysis of phospholipase A2 activity toward mixed micelles and its implications for the study of lipolytic enzymes. J. Biol. Chem. 250, 9013-9020.

37. Raneva, V., Ivanova, T., Verger, R. \& Panaiotov, I. 1995 Comparative kinetics of phospholipase action on liposomes and monolayers of phosphatidylcholine spread at the air-water interface. Colloids Surfaces B Biointerfaces 3, 357-369.

38. Berg, O. G., Yu, B. Z., Rogers, J. \& Jain, M. K. 1991 Interfacial catalysis by phospholipase A2: determination of the interfacial kinetic rate constants. Biochemistry 30, 7283-7297. (doi:10.1021/bi00243a034)

39. Jain, M. K., Yu, B. Z., Gelb, M. H. \& Berg, O. G. 1992 Assay of phospholipases A2 and their inhibitors by kinetic analysis in the scooting mode. Mediators Inflamm. 1, 85-100. (doi:10.1155/S0962935192000164)

40. Perham, M., Stagg, L. \& Wittung-Stafshede, P. 2007 Macromolecular crowding increases structural content of folded proteins. FEBS Lett. 581, 5065-5069. (doi:10.1016/j.febslet.2007.09.049)

41. Luby-Phelps, K. 2000 Cytoarchitecture and physical properties of cytoplasm: volume, viscosity, diffusion, intracellular surface area. Int. Rev. Cytol. 192, 189-221.

42. Stagg, L., Zhang, S.-Q., Cheung, M. S. \& Wittung-Stafshede, P. 2007 Molecular crowding enhances native structure and stability of alpha/beta protein flavodoxin. Proc. Natl. Acad. Sci. U. S. A. 104, 18976-18981. (doi:10.1073/pnas.0705127104)

43. Dymond, M. K. 2015 Mammalian phospholipid homeostasis: Homeoviscous adaptation deconstructed by lipidomic data driven modelling. Chem. Phys. Lipids 191, 136-146. (doi:10.1016/j.chemphyslip.2015.09.003) 
44. Dymond, M. K. 2016 Mammalian phospholipid homeostasis : evidence that membrane curvature elastic stress drives homeoviscous adaptation in vivo. J. R. Soc. Interface, 20160228.

45. Haider, A. et al. 2018 PCYT1A Regulates Phosphatidylcholine Homeostasis from the Inner Nuclear Membrane in Response to Membrane Stored Curvature Elastic Stress. Dev. Cell 45, 481-495.e8. (doi:10.1016/j.devcel.2018.04.012)

46. Hunter, J. E., Brandsma, J., Dymond, M. K., Koster, G., Mark Moore, C., Postle, A. D., Mills, R. A. \& Attard, G. S. 2018 Lipidomics of Thalassiosira pseudonana under phosphorus stress reveal underlying phospholipid substitution dynamics and novel diglycosylceramide substitutes. Appl. Environ. Microbiol. 84, 1-17. (doi:10.1128/AEM.02034-17)

47. Corsi, J., Dymond, M. K., Ces, O., Muck, J., Zink, D. \& Attard, G. S. 2008 DNA that is dispersed in the liquid crystalline phases of phospholipids is actively transcribed. Chem. Commun. (Camb). , 2307-9. (doi:10.1039/b801199k)

48. Black, C. F., Wilson, R. J., Nylander, T., Dymond, M. K. \& Attard, G. S. 2010 Linear dsDNA partitions spontaneously into the inverse hexagonal lyotropic liquid crystalline phases of phospholipids. J. Am. Chem. Soc. 132, 9728-32. (doi:10.1021/ja101550c)

49. Wilson, R. J., Tyas, S. R., Black, C. F., Dymond, M. K. \& Attard, G. S. 2010 Partitioning of ssRNA Molecules between Preformed Monolithic H(II) Liquid Crystalline Phases of Lipids and Supernatant Isotropic Phases. Biomacromolecules , 3022-3027. (doi:10.1021/bm1008469)

50. Reis, P., Holmberg, K., Watzke, H., Leser, M. E. \& Miller, R. 2009 Lipases at interfaces: A review. Adv. Colloid Interface Sci. 147-148, 237-250. (doi:10.1016/j.cis.2008.06.001)

51. Balashev, K., Jensen, T. R., Kjaer, K. \& Bjørnholm, T. 2001 Novel methods for studying lipids and lipases and their mutual interaction at interfaces. Part I. Atomic force microscopy. Biochimie 83, 387-397. (doi:10.1016/S0300-9084(01)01265-2) 
52. Jensena, T. R., Balashev, K., Bjørnholm, T. \& Kjaera, K. 2001 Novel methods for studying lipids and lipases and their mutual interacion at interfaces. Part II. Surface sensitive synchrotron X-ray scattering. Biochimie 83, 399-408.

53. Bligh, E. G. \& Dyer, W. J. 1959 A rapid method of total lipid extraction and purification. Can. J. Biochem. Physiol. 37, 911-917.

54. Pisaruka, J. \& Dymond, M. K. 2016 A low volume 3D-printed temperature-controllable cuvette for UV visible spectroscopy. Anal. Biochem. 510, 52-55.

(doi:10.1016/j.ab.2016.07.019)

55. Stewart, J. C. M. 1980 Colorimetric determination of phospholipids with ammonium ferrothiocyanate. Anal. Biochem. 104, 10-14.

56. Bradford, M. M. 1976 A rapid and sensitive method for the quantitation of microgram quantities of protein utilizing the principle of protein-dye binding. Anal. Biochem. 72, $248-254$.

57. Hoops, S., Gauges, R., Lee, C., Pahle, J., Simus, N., Singhal, M., Xu, L., Mendes, P. \& Kummer, U. 2006 COPASI - A COmplex PAthway SImulator. Bioinformatics 22, 30673074. (doi:10.1093/bioinformatics/btl485)

58. Tsaloglou, M.-N., Attard, G. S. \& Dymond, M. K. 2011 The effect of lipids on the enzymatic activity of 6-phosphofructo-1-kinase from B. stearothermophilus. Chem. Phys. Lipids 164, 713-21. (doi:10.1016/j.chemphyslip.2011.08.003)

59. Kumar, M., Mukherjee, J., Sinha, P., Sharma, S., Gupta, M. N. \& Singh, T. P. 2015 Enhancement of stability of a lipase by subjecting to three phase partitioning (TPP): structures of native and TPP-treated lipase from Thermomyces lanuginosa. Sustain. Chem. Process. 3. (doi:10.1186/s40508-015-0043-4)

60. Deems, R. A. \& Dennis, E. A. 1975 Characterization and Physical Properties of the Major Form of Phospholipase A2 from Cobra Venom (Naja naja naja) that has a molecular weight of 11,000. J. Biol. Chem 250, 9008-9012. 
61. Minton, A. P. 2001 The Influence of Macromolecular Crowding and Macromolecular Confinement on Biochemical Reactions in Physiological Media. J. Biol. Chem. 276, 1057710580. (doi:10.1074/jbc.R100005200)

62. Hou, L., Lanni, F. \& Luby-Phelps, K. 1990 Tracer diffusion in F-actin and Ficoll mixtures. Toward a model for cytoplasm. Biophys. J. 58, 31-43. (doi:10.1016/S00063495(90)82351-1)

63. Burke, J. R., Witmer, M. R. \& Tredup, J. A. 1999 The size and curvature of anionic covesicle substrate affects the catalytic action of cytosolic phospholipase A2. Arch. Biochem. Biophys. 365, 239-247. (doi:10.1006/abbi.1999.1151)

64. Middleton, E. R. \& Rhoades, E. 2010 Effects of curvature and composition on $\alpha$-synuclein binding to lipid vesicles. Biophys. J. 99, 2279-2288. (doi:10.1016/j.bpj.2010.07.056)

65. Chao, H., Martin, G. G., Russell, W. K., Waghela, S. D., Russell, D. H., Schroeder, F. \& Kier, A. B. 2002 Membrane charge and curvature determine interaction with acyl-CoA binding protein (ACBP) and fatty acyl-CoA targeting. Biochemistry 41, 10540-10553. (doi:10.1021/bi0259498)

66. Zhang, W.X., Frahm, G., Morley, S., Manor, D. and Atkinson, J. 2009 Effect of Bilayer Phospholipid Composition and Curvature on Ligand Transfer by the $\alpha$-Tocopherol Transfer Protein. Lipids 44, p.631.

67. Wilschut, J. C., Regts, W., Westernberg, H. \& Scherphof, G. 1978 Action of phospholipases $\mathrm{A} 2$ on phosphatidylcholine bilayers: effects of the phase transition temperature, bilayer curvature and structural defects. Biochem. Biophys. Acta 508, 185196.

68. Singh, J. \& Ranganathan, R. 2014 Surface dilution kinetics of phospholipase A2 catalyzed lipid-bilayer hydrolysis. J. Phys. Chem. B 118, 2077-2083. (doi:10.1021/jp411512c)

69. Wilschut, J. C., Regts, J. \& Scherphof, G. 1979 Action of Phospholipase A2 on Phospholipid Vesicles. Febs Lett. 98, 181-186. 
70. Cevc, G. \& Richardsen, H. 1999 Lipid vesicles and membrane fusion. Adv. Drug. Deliv. 38, 207-232. (doi:10.1016/S0169-409X(99)00030-7)

71. Liu, Y., Zhu, L., Yang, J., Sun, J., Zhao, J. \& Liang, D. 2015 Axial growth and fusion of liposome regulated by macromolecular crowding and confinement. Langmuir 31, 48224826. (doi:10.1021/la504699y)

72. Huang, C. 1969 Studies on phosphatidylcholine vesicles. Formation and physical characteristics. Biochemistry 8, 344-352. (doi:10.1021/bi00829a048)

73. Brouillette, C. G., Segrest, J. P., Ng, T. C. \& Jones, J. L. 1982 Minimal size phosphatidylcholine vesicles: effects of radius of curvature on head group packing and conformation. Biochemistry 21, 4569-4575. (doi:10.1021/bi00262a009)

74. Leventis, R. \& Silvius, J. R. 2010 Quantitative experimental assessment of macromolecular crowding effects at membrane surfaces. Biophys. J. 99, 2125-2133. (doi:10.1016/j.bpj.2010.07.047)

75. Kim, J. S. \& Yethiraj, A. 2010 Crowding effects on association reactions at membranes. Biophys. J. 98, 951-958. (doi:10.1016/j.bpj.2009.11.022)

76. Wang, Y., Zhao, M., Song, K., Wang, L., Tang, S. \& Riley, W. W. 2010 Partial hydrolysis of soybean oil by phospholipase A1 (Lecitase Ultra). Food Chem. 121, 1066-1072. (doi:10.1016/j.foodchem.2010.01.051) 
Supplementary Information; Macromolecular crowding and membrane binding proteins: the case of phospholipase $A_{1}$

Yuzhang Wei ${ }^{1}$, Isabel Mayoral-Delgado ${ }^{1}$, Nicolas A. Stewart ${ }^{1}$, Marcus K. Dymond ${ }^{1 *}$

${ }^{1}$ Division of Chemistry, School of Pharmacy and Biological Sciences, University of Brighton, BN2 4GL

*Author for correspondence: M.Dymond@brighton.ac.uk 
Section S1 Calibration curves for the Bradford assay [1] in the presence of Ficoll 400.

Calibration curves for the Bradford assay were constructed using $0.1 \mathrm{ml}$ of assay buffer containing protein and $3 \mathrm{ml}$ of Coomassie Brilliant Blue G-250 reagent using the standard protocol [1]. The net absorbance of a sample was calculated by subtracting the absorbance of a control sample containing no protein from each sample. Absorbance measurements were performed at $595 \mathrm{~nm}$. Samples were prepared in triplicate and data show the mean \pm standard deviation.

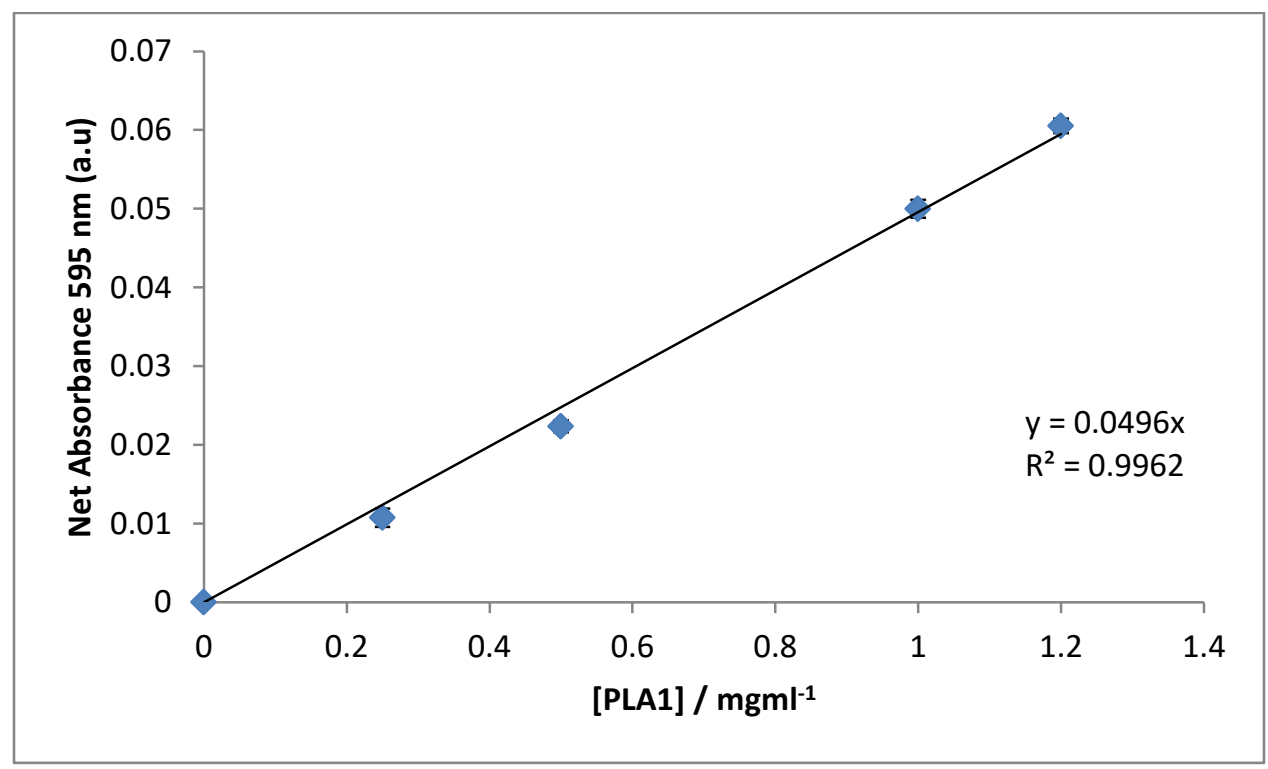

Figure S1 calibration curve for PLA 1 using the Bradford Assay in uncrowded ( 0 wt\% Ficoll) assay buffer. Data are the average of three independent repeats plus/ minus the standard deviation. Absorbance measurements were made at $595 \mathrm{~nm}$. 


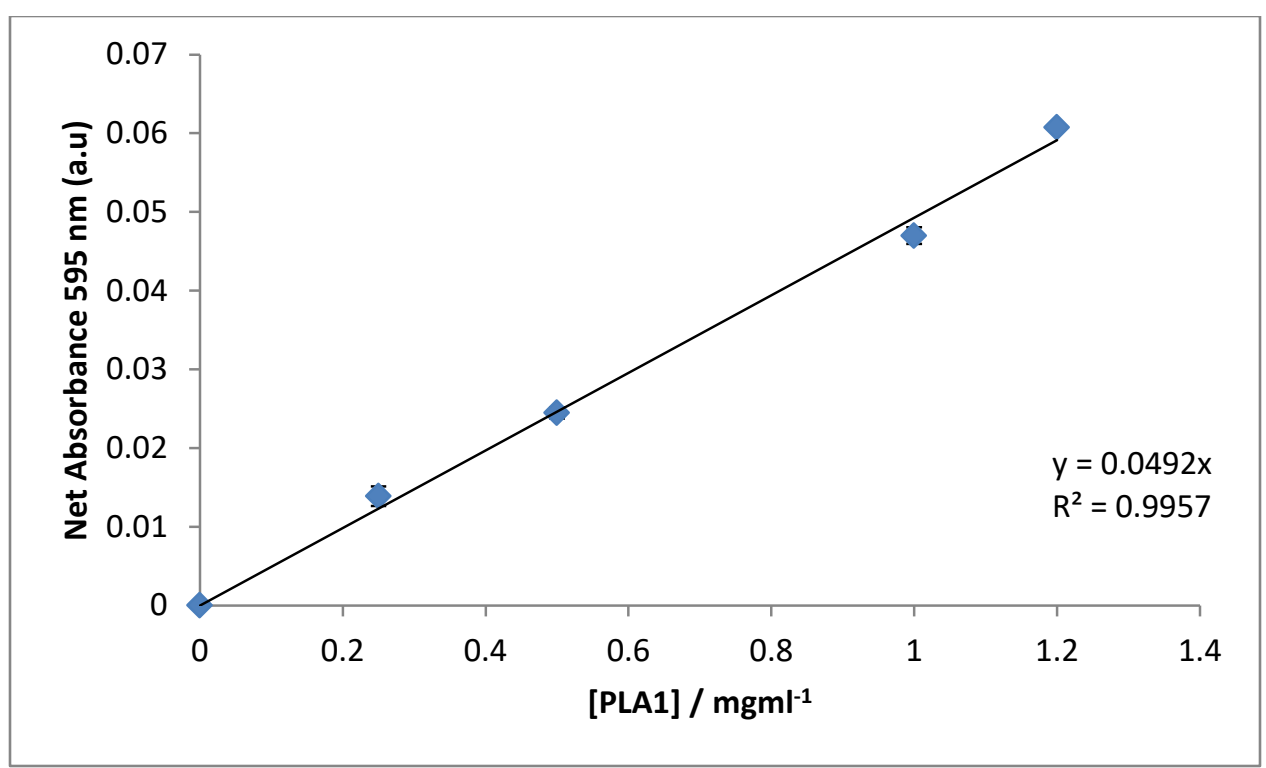

Figure S2 calibration curve for PLA 1 using the Bradford Assay in crowded (0.5wt \% Ficoll) assay buffer. Data are the average of three independent repeats plus/ minus the standard deviation. Absorbance measurements were made at $595 \mathrm{~nm}$.

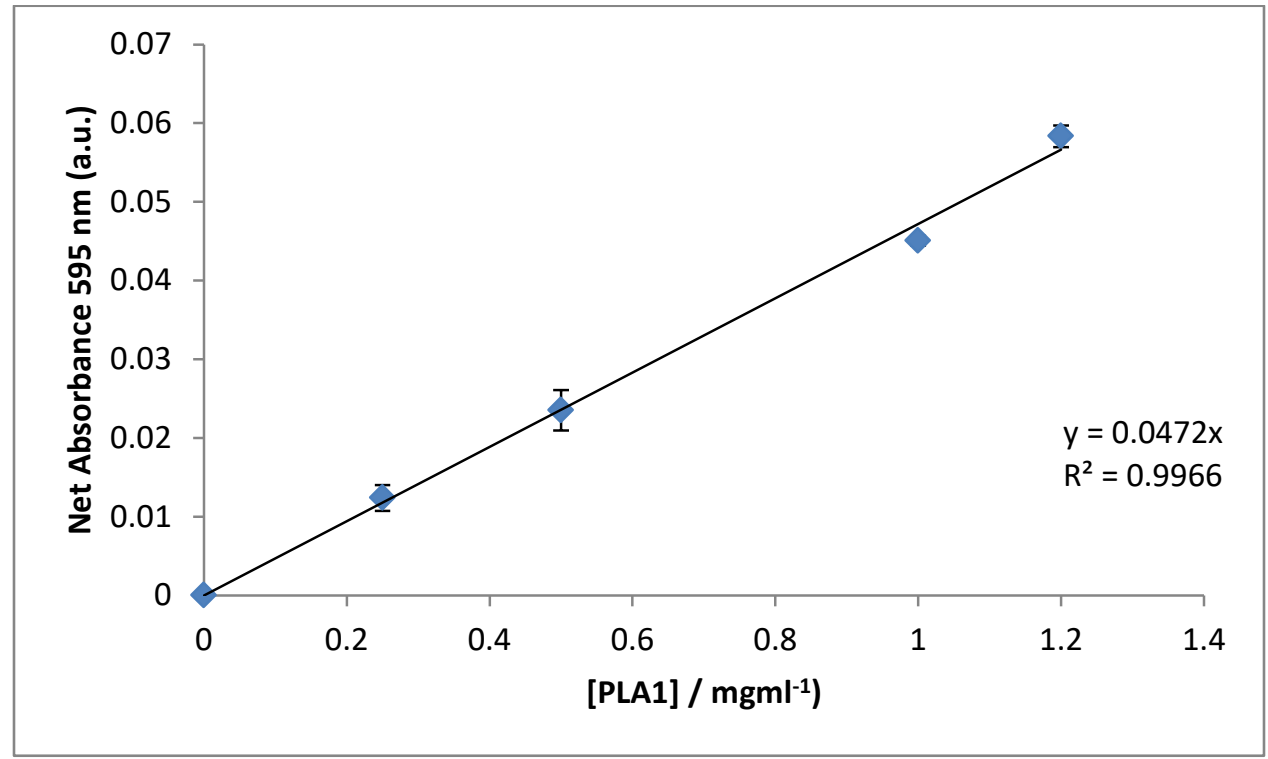

Figure S3 calibration curve for PLA $\mathrm{P}_{1}$ using the Bradford Assay in crowded (1.0 wt \% Ficoll) assay buffer. Data are the average of three independent repeats plus/ minus the standard deviation. Absorbance measurements were made at $595 \mathrm{~nm}$. 


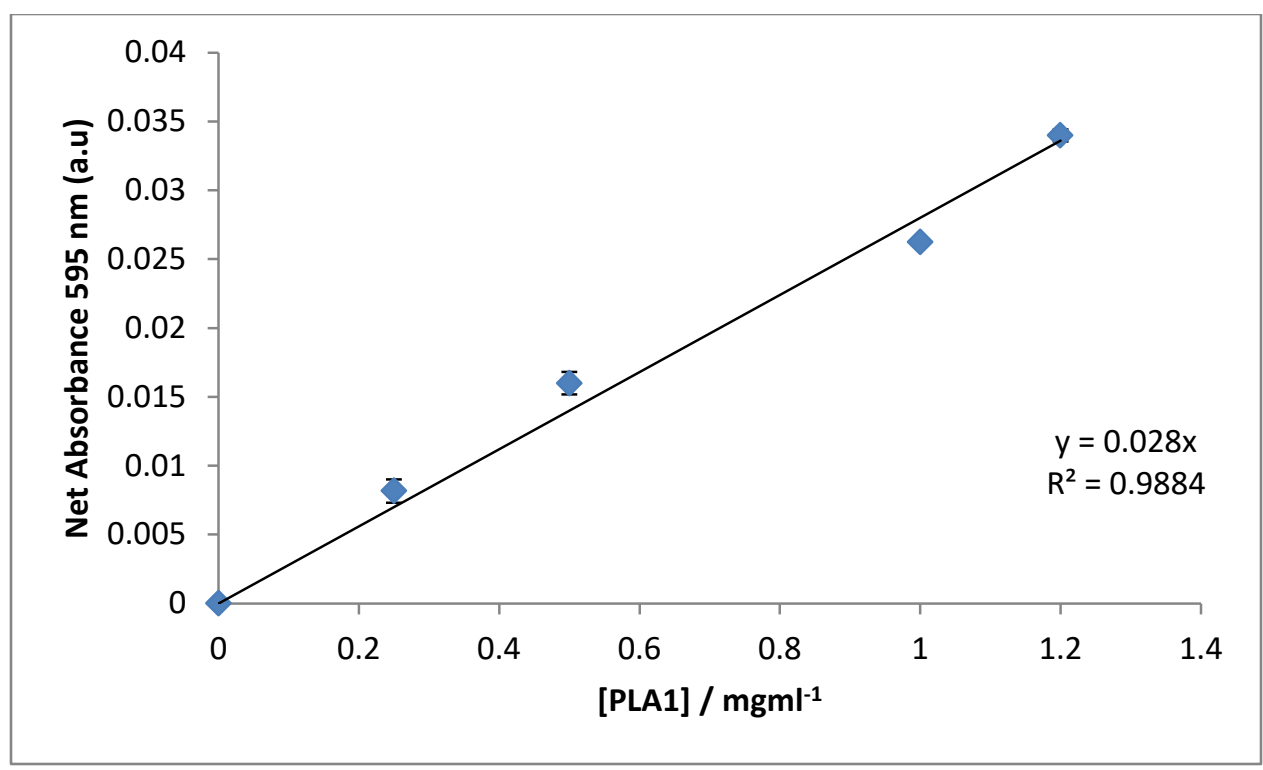

Figure S4 calibration curve for PLA 1 using the Bradford Assay in crowded (2.0 wt \% Ficoll) assay buffer. Data are the average of three independent repeats plus/ minus the standard deviation. Absorbance measurements were made at $595 \mathrm{~nm}$.

Section S2 Calibration curves for the Stewart assay of DOPC in the presence of Ficoll 400.

Calibration curves were constructed from a stock solution of $1 \mathrm{mg} / \mathrm{ml}$ DOPC in chloroform (Sigma Aldrich UK). Samples were aliquoted into centrifuge tubes and dried under vacuum. 0.5 $\mathrm{ml}$ of buffer containing $0,0.5,1$ or $2 \mathrm{wt} \%$ Ficoll 400, was then added to the dry lipids and the sample was freeze dried. $2 \mathrm{ml}$ chloroform and $2 \mathrm{ml}$ ammonium ferrithiocyanate solution was then added, centrifuged and separated according to the standard Stewart assay protocol [2]. Absorbance was measured at $485 \mathrm{~nm}$. Samples were prepared in triplicate and data show the mean average \pm standard deviation. 


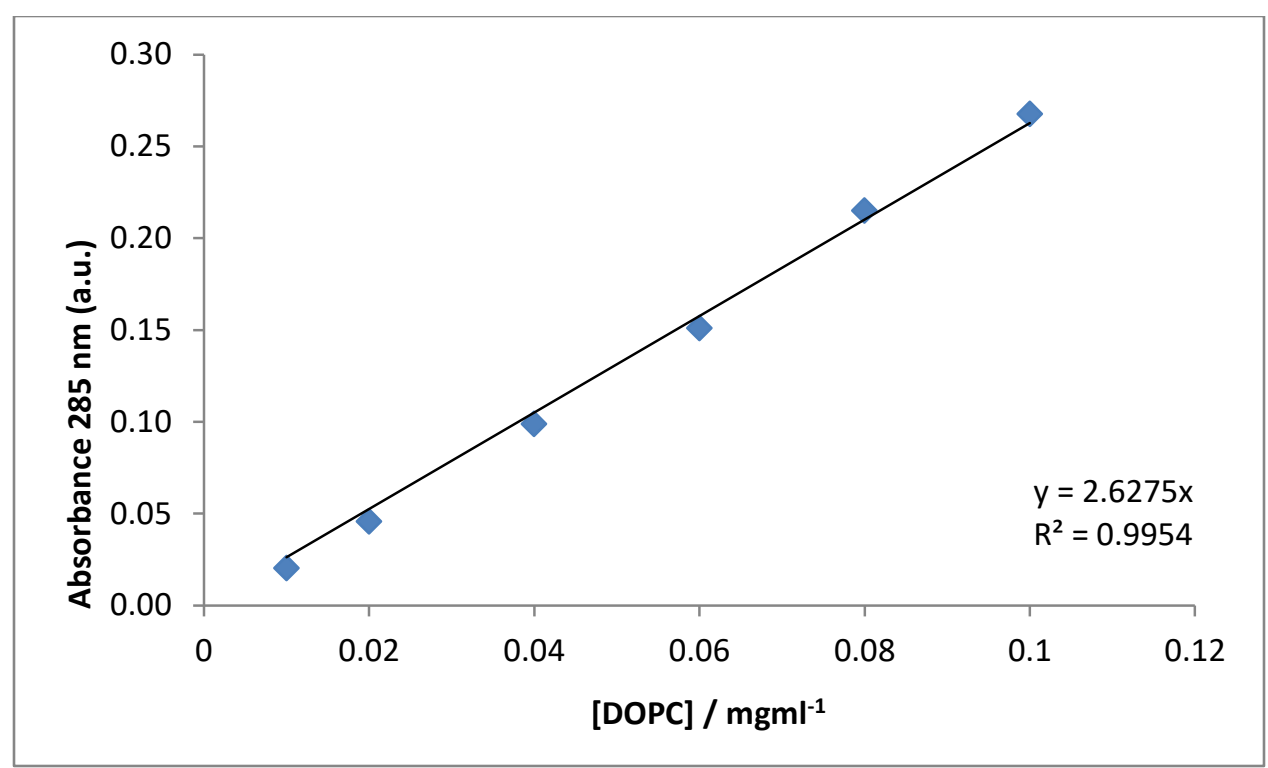

Figure S5 calibration curve for DOPC using the Stewart Assay in crowded (0 wt \% Ficoll) assay buffer. Data are the average of three independent repeats plus/ minus the standard deviation. Absorbance measurements were made at $485 \mathrm{~nm}$.

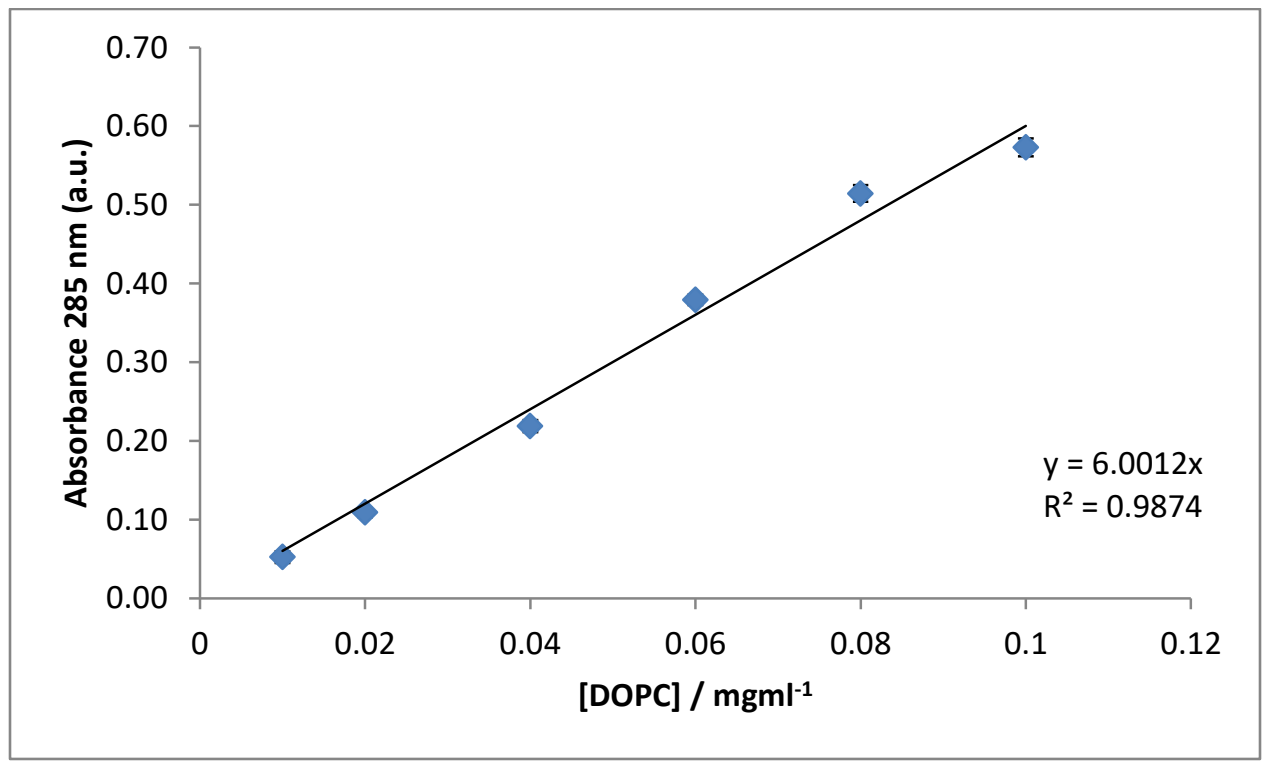

Figure S6 calibration curve for DOPC using the Stewart Assay in crowded (0.5 wt \% Ficoll) assay buffer. Data are the average of three independent repeats plus/ minus the standard deviation. Absorbance measurements were made at $485 \mathrm{~nm}$. 


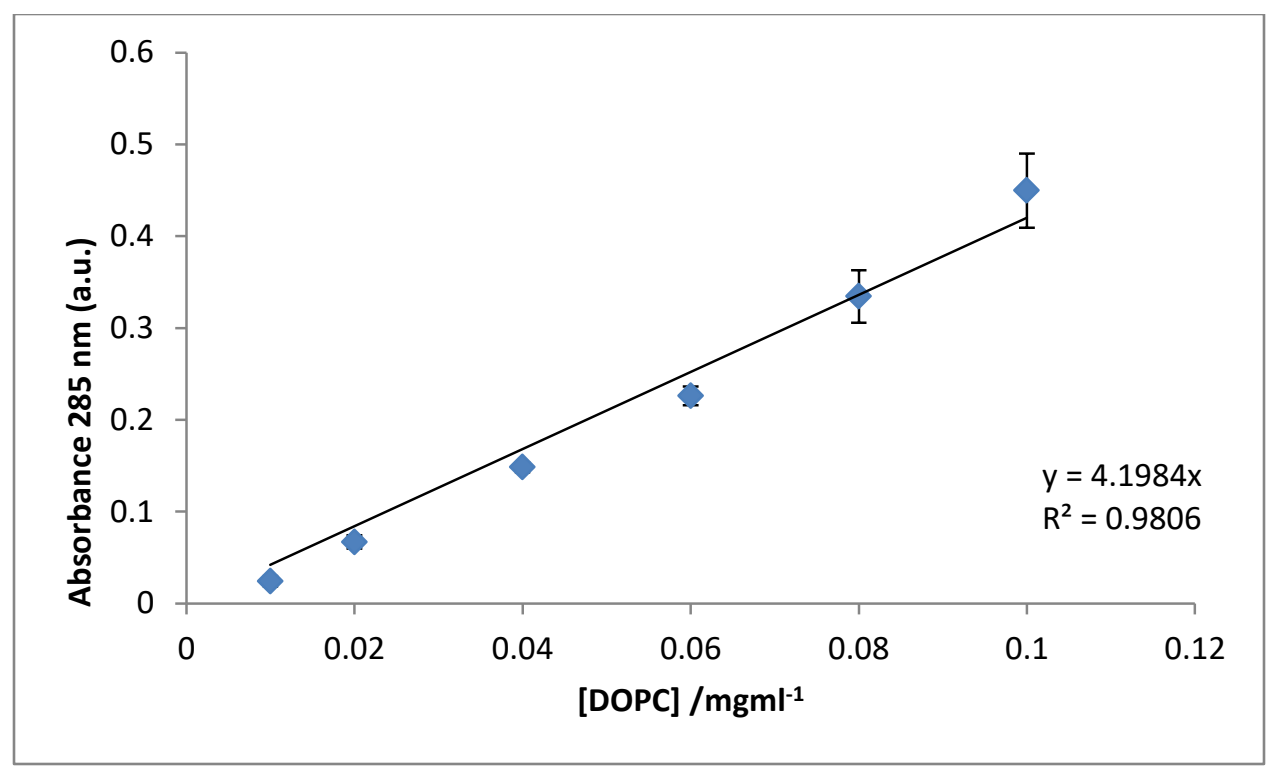

Figure S7 calibration curve for DOPC using the Stewart Assay in crowded (1 wt \% Ficoll) assay buffer. Data are the average of three independent repeats plus/ minus the standard deviation. Absorbance measurements were made at $485 \mathrm{~nm}$.

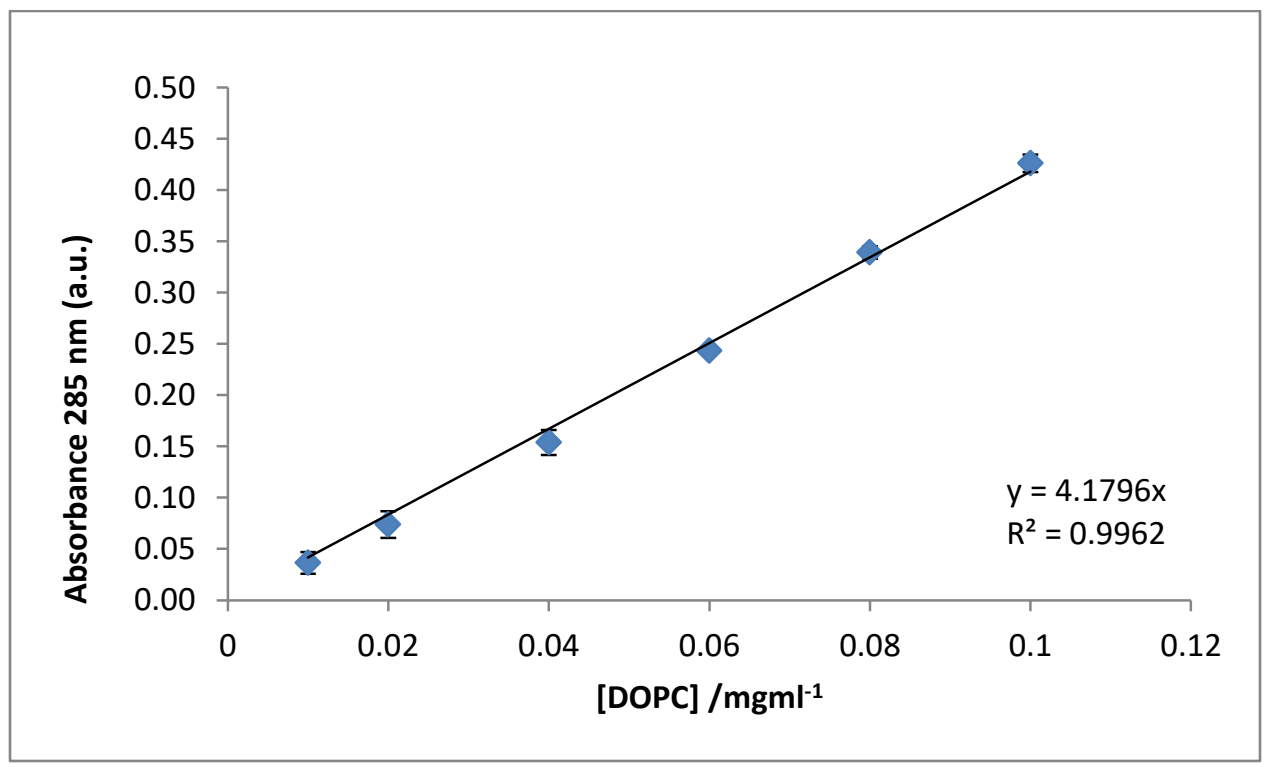

Figure S8 calibration curve for DOPC using the Stewart Assay in crowded ( 2 wt \% Ficoll) assay buffer. Data are the average of three independent repeats plus/ minus the standard deviation. Absorbance measurements were made at $485 \mathrm{~nm}$. 
Section S3 Non-specific binding studies of PLA1 and DOPC to 3D-prints made from polylactic acid

Non-specific binding studies were carried out in 3D-printed 'sample tubes', see Figure S8.

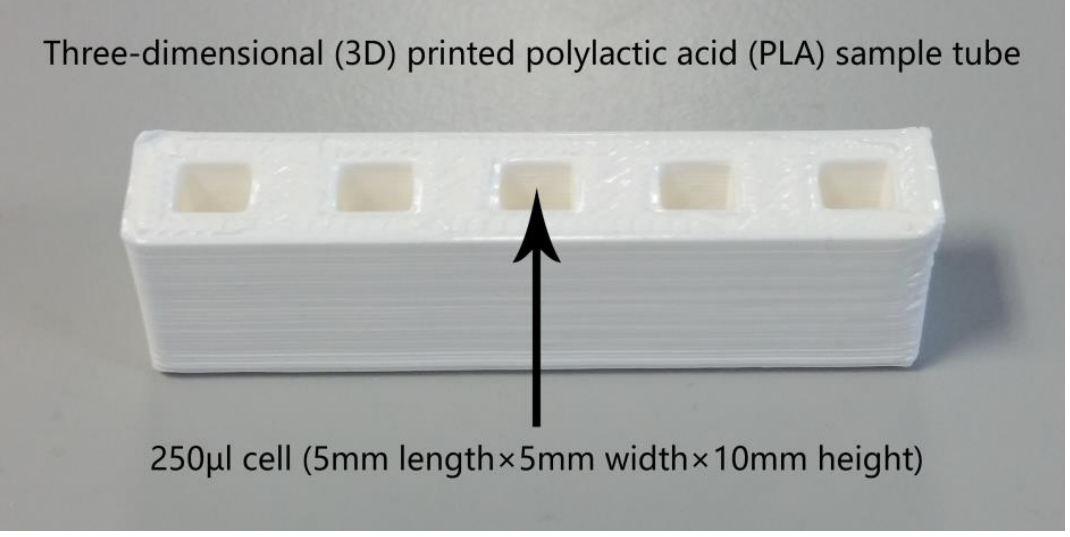

Figure S9 A bespoke3D-printed sample tube with capacity of $250 \mu \mathrm{l}(5 \mathrm{~mm} \times 5 \mathrm{~mm} \times 10 \mathrm{~mm}$ ) and an internal surface area of $0.25 \mathrm{~cm}^{2}$.

Samples of $200 \mu$ l containing either DOPC vesicles $(0.9 \mathrm{mg} / \mathrm{ml})$ or PLA1 $(0.27 \mathrm{mg} / \mathrm{ml})$ dissolved in $0 \mathrm{wt} \%$ buffer were placed in the sample tube and incubated at room temperature. Measurements were performed in triplicate and after 60,120 or 240 minutes, $100 \mu$ l of sample was removed and the concentration of DOPC or PLA 1 was quantified by either the Bradford or Stewart assay, methods shown in section S1 and S2. Figure S10 shows the variation of DOPC concentration w.r.t. time, which demonstrates that there is negligible non-specific binding of DOPC to the polylactic acid 3D print. Figure S11 shows the variation of PLA $A_{1}$ concentration w.r.t. time, which demonstrates that there is a significant amount of non-specific binding of $\mathrm{PLA}_{1}$ to the polylactic acid 3D print. 


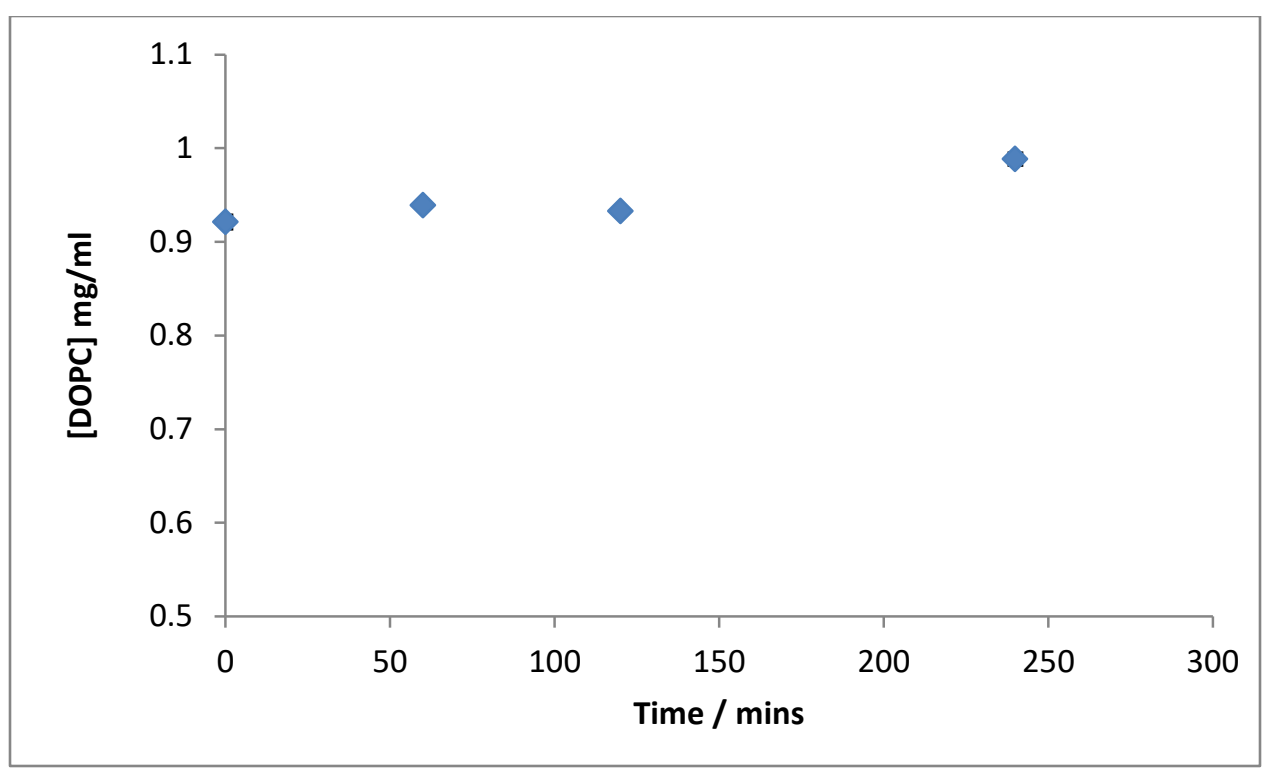

Figure S10 the concentration of DOPC after incubation in the 3D-printed sample tubes shown in Figure S9. The data show negligible binding of DOPC vesicles ( $0 \mathrm{wt} \%$ buffer) to the polylactic acid sample tube, after 4 hours. Data are the average of three independent repeats plus/ minus the standard deviation. Note error bars are obscured by the symbols.

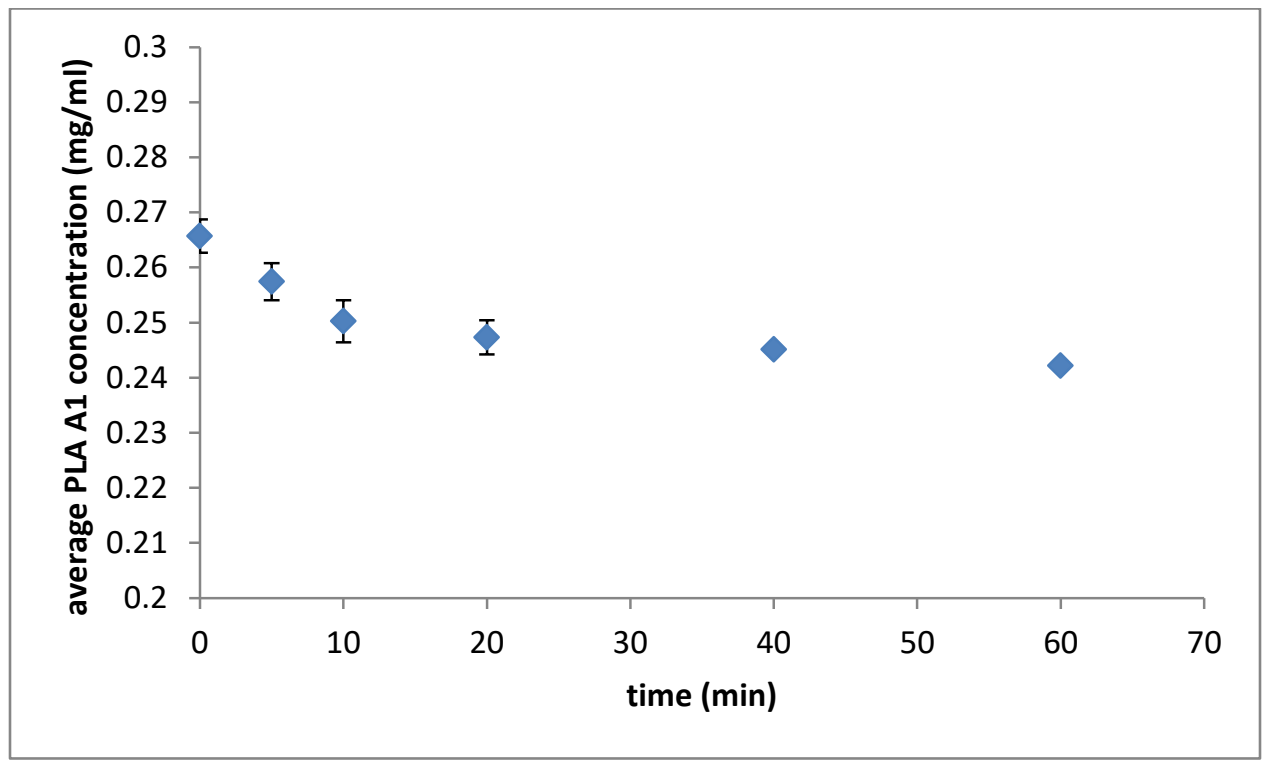

Figure S11 the concentration of $\mathrm{PLA}_{1}$ after incubation in the 3D-printed sample tubes shown in Figure S9. The data show that some binding $(0.024 \mathrm{mg})$ of $\mathrm{PLA}_{1}$ to the polylactic acid sample tube occurs, although this equilibrates after 60 minutes. The surface area of the sample tube is $2 \mathrm{~cm}^{2}$ giving $0.012 \mathrm{mg} / \mathrm{cm}^{2}$ of binding of $\mathrm{PLA}_{1}$ to polylactic acid. Data are the average of three independent repeats plus/ minus the standard deviation. 
Our results showed that $\mathrm{PLA}_{1}$ does bind non-specifically to polylactic acid. From a starting concentration of $0.26 \mathrm{mg} / \mathrm{ml} \mathrm{PLA}_{1}$, taking about 1 hour to equilibrate, $0.024 \mathrm{mg}$ of PLA $\mathrm{A}_{1}$ was adsorbed from solution. The internal surface area of our 3D-printed chamber was $2 \mathrm{~cm}^{2}$, suggesting that $0.012 \mathrm{mg} / \mathrm{cm}^{2}$ of $\mathrm{PLA}_{1}$ bind to the internal polylactic acid surface.

Section S4 Non-specific binding studies of PLA $A_{1}$ and DOPC to 3D-printed equilibrium dialysis chambers

S4.1 Control studies: vesicles do not cross the dialysis membrane or bind non-specifically to the 3D-printed device

Next we assessed if DOPC vesicles would cross the dialysis membrane in the 3D-printed equilibrium dialysis device, we placed 0 wt\% buffer $(1 \mathrm{ml})$ into chamber $A$ and 0 wt\% buffer ( $1 \mathrm{ml}$ containing DOPC vesicles) into chamber $B$, after $0.5,1$ and 2 hours we quantified the amount of DOPC in both chamber $A$ and $B$. We found that the DOPC concentration in chamber $B$ was unchanged from the starting DOPC concentration of $0.9 \mathrm{mg} / \mathrm{ml}$. No significant quantities of lipid were detected in chamber A over the same time period. This finding also demonstrates that there is negligible non-specific binding of DOPC vesicles to the dialysis membrane. We note that whilst DOPC monomers can cross the membrane, the critical aggregation concentration of DOPC is too low (circa $1 \times 10^{-12} \mathrm{M}$ ) to enable vesicles to form and bind $\mathrm{PLA}_{1}$ in chamber $A$. 


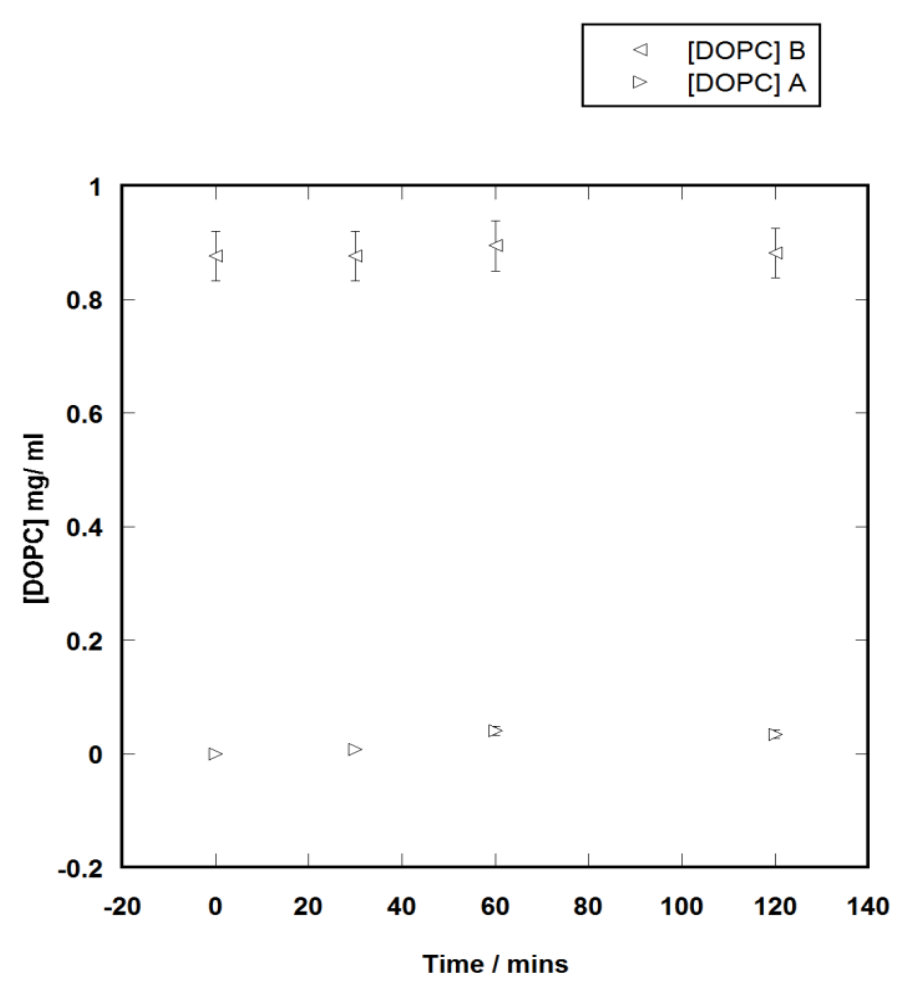

Figure S12 the behaviour of DOPC vesicles in the 3D-printed equilibrium dialysis system fitted with $50 \mathrm{kD}$ dialysis membrane, showing that DOPC vesicles do not cross from chamber $A$ to chamber B, over the experimental timescale. Data are the average of three independent repeats plus/ minus the standard deviation.

S4.2 Control studies: PLA 1 crosses the dialysis membrane and binds non-specifically to the 3D-print material polylactic acid

The internal surface area of the 3D-printed equilibrium dialysis device in Figure 1 is up to $8 \mathrm{~cm}^{2}$, depending on the volume of liquid used, suggesting that the non-specific binding of PLA to the polylactic acid could cause challenges when quantifying the binding of PLA 1 to DOPC vesicles, unless accounted for.

We investigated $\mathrm{PLA}_{1}$ crossing the dialysis membrane in the absence of lipid vesicles.

The experimental set-up in Figure 1 was designed so that each chamber, A or $B$, has an identical volume and thus, assuming free passage of $\mathrm{PLA}_{1}$, there will be equal concentrations of the PLA in both chambers at equilibrium. If there is negligible non-specific binding of $\mathrm{PLA}_{1}$ the equilibrium concentration will be half the initial concentration. Figure $S 13$, shows the concentration of $\mathrm{PLA}_{1}$ as determined in both chamber $A$ and chamber $B$ at different intervals of time. 

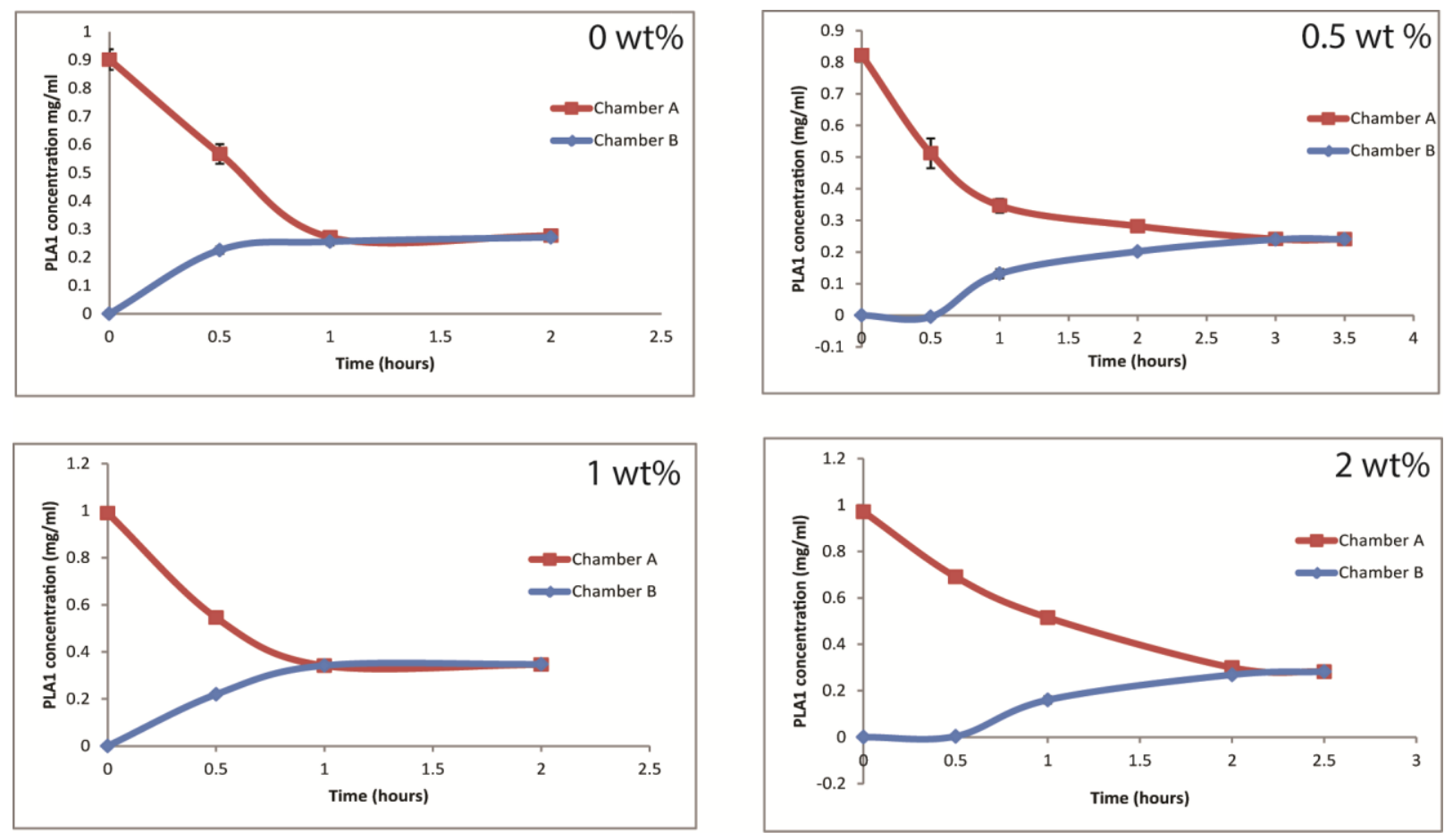

Figure S13 Full analysis of the passage of $\mathrm{PLA}_{1}$ through equilibrium dialysis system in buffers with different wt\% Ficoll 400. The data clearly show that at all wt \% Ficoll $400 \mathrm{PLA}_{1}$ passes from chamber $A$ through the dialysis membrane to chamber $B$. Data are the average of three independent repeats plus/ minus the standard deviation.

There are two key features in Figure S13; the first is that at equilibrium the amount of $\mathrm{PLA}_{1}$ in solution in both chambers is equal at all compositions of crowding agent, which confirms that $\mathrm{PLA}_{1}$ can cross the dialysis membrane. The second observation is that more than half the $\mathrm{PLA}_{1}$ has disappeared from solution. This suggests that as well as crossing the membrane there is a significant degree of non-specific binding, up to $40 \%$, of the PLA $A_{1}$ in the system. Some of this can be accounted for by taking account of the non-specific binding of PLA to the polylactic acid 3D-print.

The internal surface area of the filled $(2 \mathrm{ml}) 3 \mathrm{D}$-printed equilibrium dialysis cell is $8 \mathrm{~cm}^{2}$ therefore up to $0.1 \mathrm{mg}$ (based upon polylactic acid binding around $0.012 \mathrm{mg} / \mathrm{cm}^{2}$ of PLA $_{1}$ ) binds non-specifically to the polylactic acid. Thus from a typical starting concentration of $1 \mathrm{mg} / \mathrm{ml}$ (as shown in Figure S13) $0.5 \mathrm{mg}$ binds to, or is in the process of diffusion through, the dialysis membrane.

It should be noted that, whilst the time taken for $\mathrm{PLA}_{1}$ to reach an equilibrium concentration in chamber B increases from 0 wt\% Ficoll 400 (1 hour) to 2 wt\% Ficoll 400 (2.5 
hours), we believe that the amount of non-specific binding in the system (greater than half the initial PLA $\mathrm{A}_{1}$ concentration) prohibits any reliable interpretation from Figure 2 of the kinetics of $\mathrm{PLA}_{1}$ diffusion in the different wt\% crowded buffers. With these observations in hand we designed an experiment to assess the extent to which different wt\% of Ficoll 400 influenced the membrane binding of $\mathrm{PLA}_{1}$ to DOPC vesicles.

S4.3 Equations and further results from the COPASI kinetic equilibrium model 
- $\frac{d[V] V_{B}}{d t}=-V_{B}\left(\left(k_{1}\left[P L A_{1 B}\right][V]-k_{-1}\left[P L A_{1 B} V\right]\right)\right)$

- $\frac{d\left[P L A_{1 A}\right] V_{A}}{d t}=-\left(\left(k_{a}\left[P L A_{1 A}\right]-k_{d}\left[P L A_{1 B}\right]\right.\right.$

- $\frac{d\left[P L A_{1 B}\right] V_{B}}{d t}=$ $+\left(\left(k_{a}\left[P L A_{1 A}\right]-k_{d}\left[P L A_{1 B}\right]\right)\right)-V_{B}\left(\left(k_{1}\left[P L A_{1 B}\right][V]-k_{-1}\left[P L A_{1 B} V\right]\right)\right)$

- $\frac{d\left[P L A_{1 V} B\right] V_{B}}{d t}=+V_{B}\left(\left(k_{1}\left[P L A_{1 B}\right][V]-k_{-1}\left[P L A_{1 B} V\right]\right)\right)$

Figure S14 Differential equations generated by COPASI for the equilibrium binding reaction scheme used to fit data to Figure 2 . All terms are defined in the main document.

- $\frac{d[D O P C] V_{B}}{d t}=-V_{B}\left(\left(k_{2}\left[P L A_{1 B} V\right][D O P C]-k_{-2}\left[P L A_{1 B} V(D O P C)\right]\right)\right)$

- $\frac{d[O A] V_{B}}{d t}=+V_{B}\left(\left(k_{3}\left[P L A_{1 B} V(D O P C)\right]-k_{-3}\left[P L A_{1 B} V\right][O A][O P C]\right)\right)$

- $\frac{d[V] V_{B}}{d t}=-V_{B}\left(\left(k_{1}\left[P L A_{1 B}\right][V]-k_{-1}\left[P L A_{1 B} V\right]\right)\right)$

- $\frac{d\left[P L A_{1 B}\right] V_{B}}{d t}=-V_{B}\left(\left(k_{1}\left[P L A_{1 B}\right][V]-k_{-1}\left[P L A_{1 B} V\right]\right)\right)$

- $\frac{d\left[P L A_{1 B} V\right] V_{B}}{d t}=+V_{B}\left(\left(k_{1}\left[P L A_{1 B}\right][V]-k_{-1}\left[P L A_{1 B} V\right]-V_{B}\left(\left(k_{2}\left[P L A_{1 B} V\right][D O P C]-\right.\right.\right.\right.$ $k_{-2}\left[P L A_{1 B} V(D O P C)\right]+V_{B}\left(\left(k_{3}\left[P L A_{1 B} V(D O P C)\right]-k_{-3}\left[P L A_{1 B} V\right][O A][O P C]\right)\right)$

- $\frac{d\left[P L A_{1 B} V(D O P C)\right]\left[V_{B}\right]}{d t}=+V_{B}\left(\left(k_{2}\left[P L A_{1 B} V\right][D O P C]-k_{-2}\left[P L A_{1 B} V(D O P C)\right]\right)\right)-$ $V_{B}\left(\left(k_{3}\left[P L A_{1 B} V(D O P C)\right]-k_{-3}\left[P L A_{1 B} V\right][O A][O P C]\right)\right)$

- $\frac{d[O P C]\left[V_{B}\right]}{d t}=+V_{B}\left(\left(k_{3}\left[P L A_{1 B} V(D O P C)\right]-k_{-3}\left[P L A_{1 B} V\right][O A][O P C]\right)\right)$

Figure S15 Differential equations generated by COPASI for the equilibrium binding reaction scheme used to fit data to Figure 3. All terms are defined in the main document. 


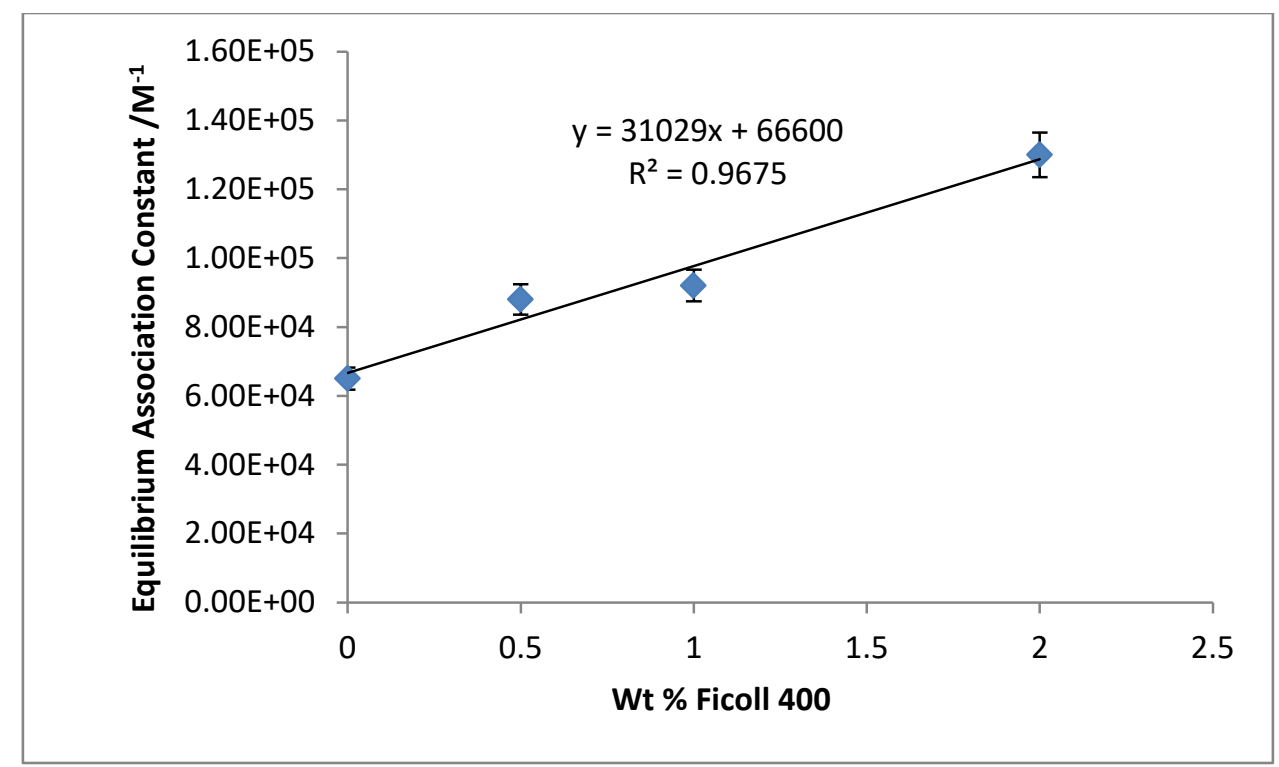

Figure S16 the change in Equilibrium association constant of PLA $\mathrm{A}_{1}$ with DOPC vesicles as wt\% Ficoll 400 and macromolecular crowding increases, error bars show a standard error of $\pm 5 \%$, which arises from the accuracy of estimate the number of available binding sites on the lipid vesicle $[\mathrm{V}]$, see main document.

Section S5 DSC and DLS studies of PLA1 in macromolecularly crowded media
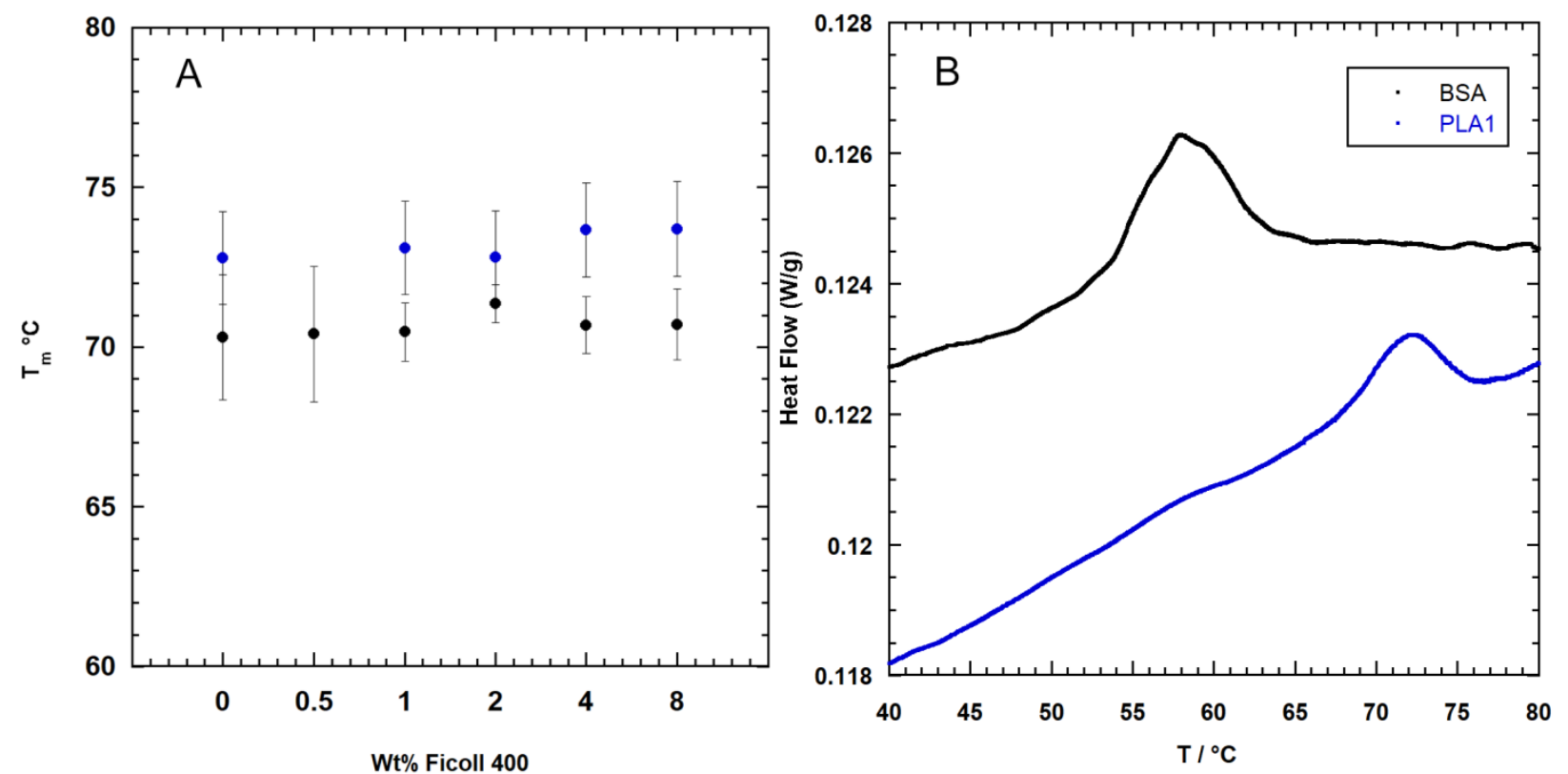

Figure $\mathrm{S} 17 \mathrm{~T}_{\mathrm{m}}$ data obtained by DSC for PLA $\mathrm{A}_{1}$ in the presence of Ficoll 400. Figure S17A shows no change within error for the $T_{m}$ of $P L A_{1}$ in $0.1 \mathrm{M}$ phosphate buffer $(\mathrm{pH} 7.2)$ black circles or 50 mM PIPES ( $\mathrm{pH} \mathrm{7.2,} 100 \mathrm{mM} \mathrm{CaCl} 240 \mathrm{mM} \mathrm{NaCl}$ ), blue circles. Error bars are the standard 
deviation of 3 repeats. Figure S17B shows an example DSC trace for $\mathrm{PLA}_{1}$ and the reference DSC trace for $B S A$, which we observed to have a $T_{m}$ of $59{ }^{\circ} \mathrm{C}$, which agrees well with values reported in the literature [3-5].
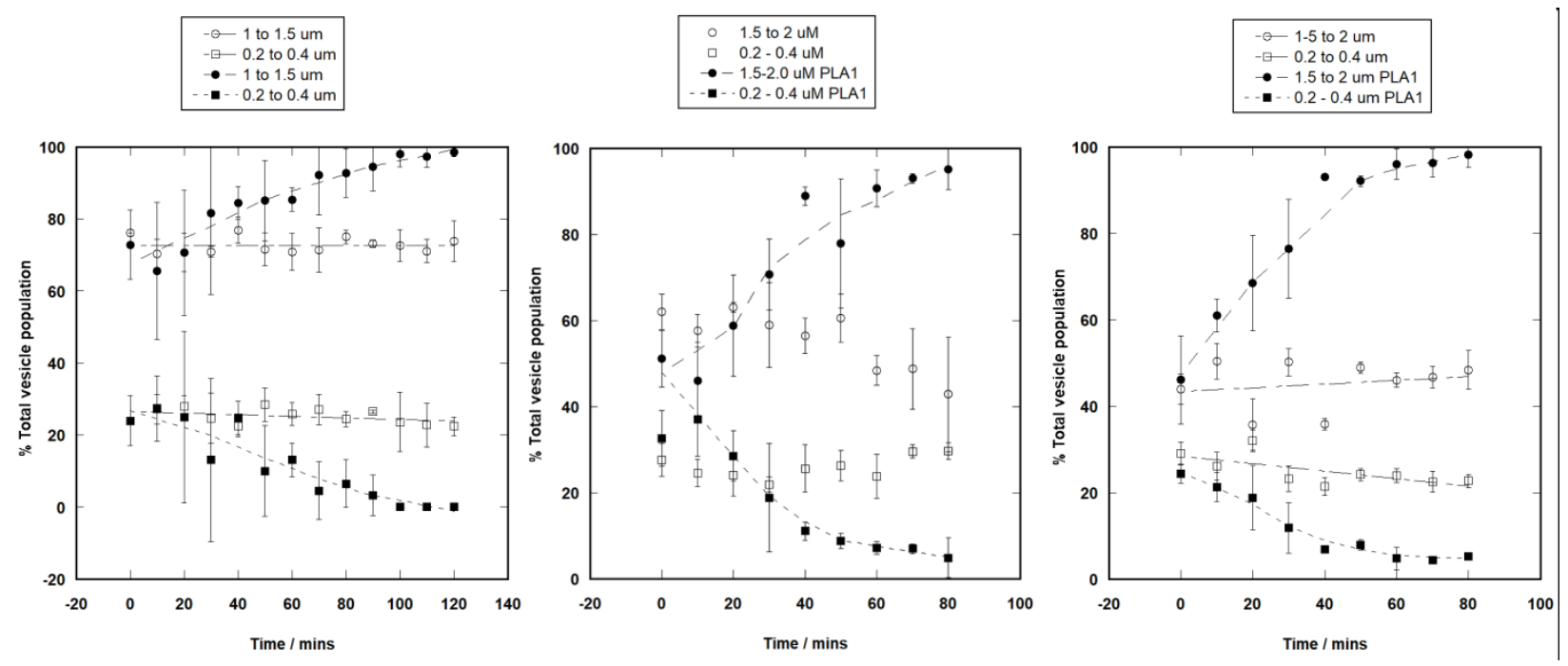

Figure S18 The time-dependent behaviour of different sized populations of vesicles treated with $\mathrm{PLA}_{1}$ at different wt\% of Ficoll 400. Figures S18A, S18B and S18C show 0, 1 and 2 wt\% Ficoll 400 respectively. Circles show vesicles in the size range of 1 to $2 \mu \mathrm{m}$ diameter. Squares show vesicles in the size range 0.2 to $0.4 \mu \mathrm{m}$. Unfilled shapes show data for vesicles in the absence of $\mathrm{PLA}_{1}$ and filled shapes show data in the presence of PLA ${ }_{1}$. Data show that the action of PLA $A_{1}$ with increasing wt\% of Ficoll 400, decreases the number of smaller vesicles in solution more rapidly. Data points are the average of three independent repeats plus/ minus the standard deviation.

Table S1 Constants used to fit sizing data shown in Figure $4 \mathrm{~b}$ using a $3^{\text {rd }}$ order polynomial of the form $y=a x^{3}+b x^{2}+c x+d$

$\begin{array}{llll}w t \% & 0 & 1 & 2\end{array}$ 


$\begin{array}{lrrr}\text { a } & -0.0027 & -0.0041 & -0.0073 \\ \text { b } & 0.28 & 0.41 & 0.64 \\ \text { c } & 1.9 & 6.7 & 12.6 \\ \text { d } & 100 & 250 & 350\end{array}$

Section S6 Data relating to assumptions in the models

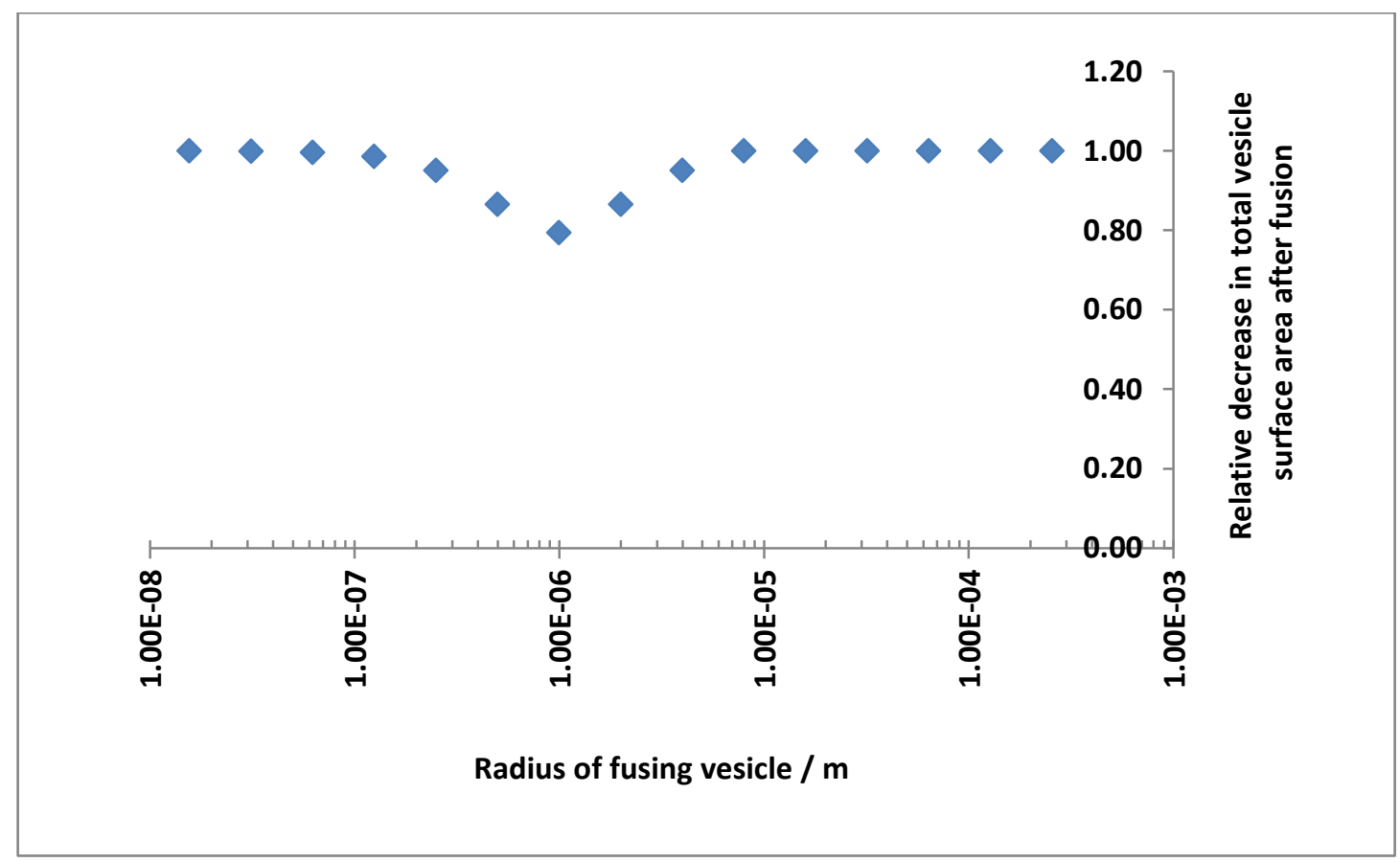

Figure S19 The relative decrease in total vesicle surface area after the fusion of two lipid vesicles. Estimates, assume to total amount of lipid is unchanged and none of the internal contents of the fusing vesicles are lost. Calculations are shown for a vesicle of radius $1 \times 10^{-6} \mathrm{~m}$ fusing with another single vesicle in the size range of $1 \times 10^{-8}$ to $1 \times 10^{-3} \mathrm{~m}$, as shown on the $\mathrm{x}$ axis. 


\section{S6.1 estimate of the stored elastic energy in DOPC vesicles}

Stored elastic energies of vesicles can be determined using the Helfrich Hamiltonian [6] shown in Equation S1. Where the stored elastic energy per amphiphile in a monolayer $\left(g_{\mathrm{c}}\right)$ is given by

$$
g_{\mathrm{c}}=1 / 2 \kappa A\left(c_{1}+c_{2}-2 c_{0}\right)^{2}+\kappa_{\mathrm{G}} A c_{1} c_{2} \quad \text { Equation S1 }
$$

Where $A$ is the cross-sectional area per molecule, $c_{1}$ and $c_{2}$ are the principal curvatures at the interface (with the convention that an interface with negative curvature curves towards water), $c_{0}$ is the intrinsic (or spontaneous) curvature of the monolayer, $k$ is the bending rigidity and $\kappa_{\mathrm{G}}$ is the Gaussian curvature modulus.

$c_{0}$ for a mixture of amphiphiles can be determined using the principle of ideal mixing such that $c_{0}$ mix is the sum of $c_{0}$ values for individual lipids multiplied by their mole fraction in the membrane [7]

We estimated the total curvature elastic energy per amphiphile for a several different compositions of vesicles to mimic the action of PLA $A_{1}$ on DOPC. This estimate assumed that $K_{G}$ and $K_{m}$ are independent of lipid composition so values for pure DOPC $\left(6.10 \times 10^{-19} \mathrm{~J}\right.$ and $0.4 \mathrm{x}$ $10^{-19} \mathrm{~J}$ [8] respectively, where $\mathrm{K}_{\mathrm{G}} / \mathrm{K}_{\mathrm{M}}=0.85$ [9]) were used throughout. The spontaneous curvature of OA was taken as 1/-11 nm [10], DOPC 1/-143 nm [11] and OPC was 1/400 nm [12]. The results of these estimates are shown in Figure S18, which clearly shows that as the mole fraction of DOPC decreases stored elastic energy in the vesicles increases, regardless of the size of vesicles. 


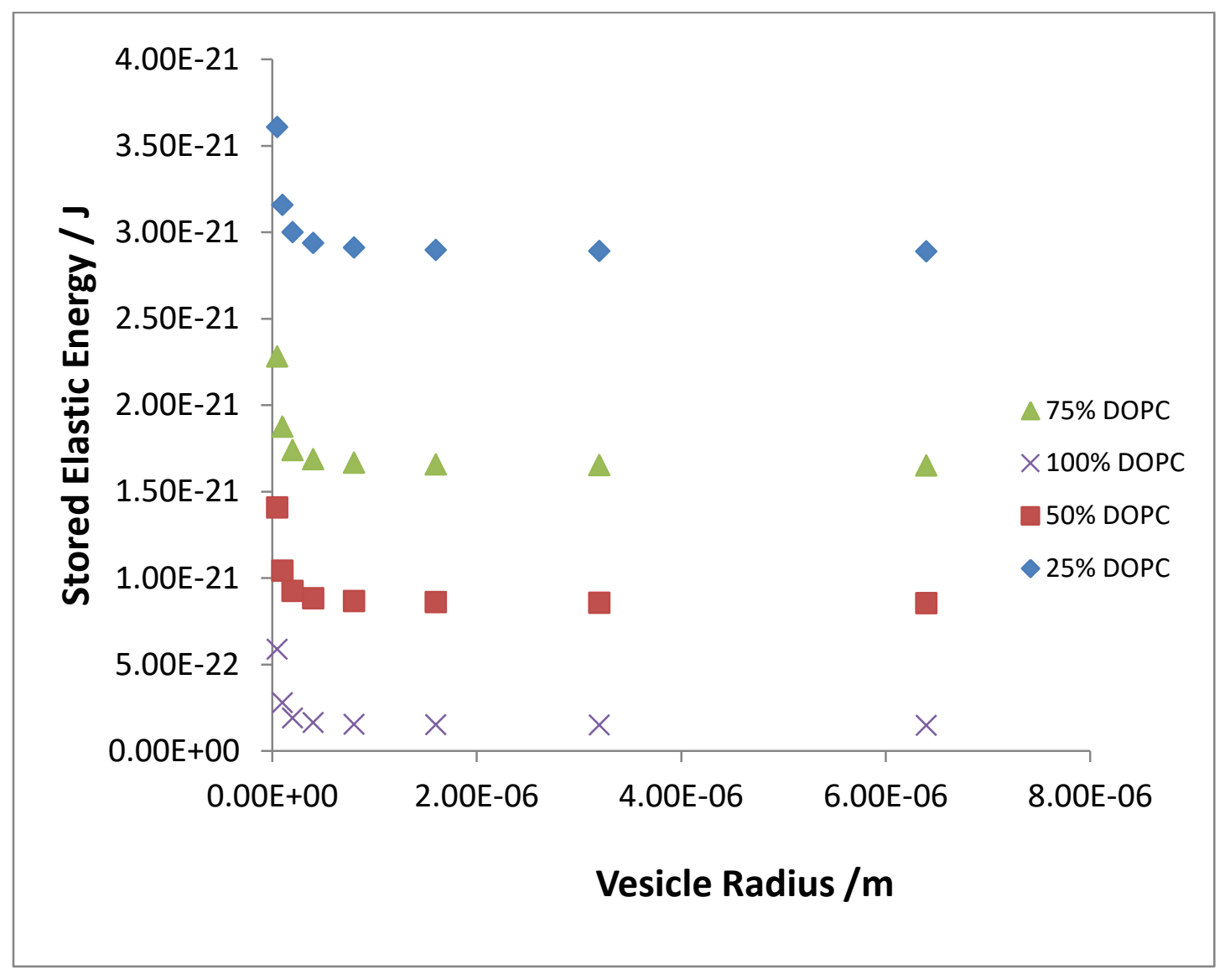

Figure S20 the range of stored elastic energies estimated for DOPC vesicles undergoing conversion to OPA and OA as catalysed by $\mathrm{PLA}_{1}$. 


\section{References}

[1] M.M. Bradford, A rapid and sensitive method for the quantitation of microgram quantities of protein utilizing the principle of protein-dye binding, Anal. Biochem. 72 (1976) 248-254.

[2] J.C.M. Stewart, Colorimetric determination of phospholipids with ammonium ferrothiocyanate, Anal. Biochem. 104 (1980) 10-14.

[3] S. Deep, J.C. Ahluwalia, Interaction of bovine serum albumin with anionic surfactants, Phys. Chem. Chem. Phys. 3 (2001) 4583-4591.

[4] A. Michnik, THERMAL STABILITY OF BOVINE SERUM ALBUMIN DSC study, J. Therm. Anal. 71 (2003) 509-519. doi:10.1023/A:1022851809481.

[5] V.A. Borzova, K.A. Markossian, N.A. Chebotareva, S.Y. Kleymenov, N.B. Poliansky, K.O. Muranov, V.A. Stein-Margolina, V. V. Shubin, D.I. Markov, B.I. Kurganov, Kinetics of Thermal Denaturation and Aggregation of Bovine Serum Albumin, PLoS One. 11 (2016) e0153495. doi:10.1371/journal.pone.0153495.

[6] W. Helfrich, Elastic properties of lipid bilayers: theory and possible experiments., Z. Naturforsch. C. 28 (1973) 693-703. doi:10.1002/mus.880040211.

[7] M.K. Dymond, R.J. Gillams, D.J. Parker, J. Burrell, A. Labrador, T. Nylander, G.S. Attard, Lipid Spontaneous Curvatures Estimated from Temperature-Dependent Changes in Inverse Hexagonal Phase Lattice Parameters: Effects of Metal Cations, Langmuir. 32 (2016) 10083-10092. doi:10.1021/acs.langmuir.6b03098.

[8] D. Marsh, Elastic curvature constants of lipid monolayers and bilayers., Chem. Phys. Lipids. 144 (2006) 146-159. doi:10.1016/j.chemphyslip.2006.08.004.

[9] M. Hu, J.J. Briguglio, M. Deserno, Determining the Gaussian curvature modulus of lipid membranes in simulations, Biophys. J. 102 (2012) 1403-1410.

doi:10.1016/j.bpj.2012.02.013.

[10] R.J. Gillams, T. Nylander, T.S. Plivelic, M.K. Dymond, G.S. Attard, Formation of inverse topology lyotropic phases in dioleoylphosphatidylcholine/oleic Acid and dioleoylphosphatidylethanolamine/oleic Acid binary mixtures., Langmuir. 30 (2014) 3337-44. doi:10.1021/la404275u.

[11] J.A. Szule, N.L. Fuller, R.P. Rand, The effects of acyl chain length and saturation of diacylglycerols and phosphatidylcholines on membrane monolayer curvature., Biophys. J. 83 (2002) 977-984.

http://www.pubmedcentral.nih.gov/articlerender.fcgi?artid=1302201\&tool=pmcentrez\& rendertype=abstract.

[12] N. Fuller, R.P. Rand, The influence of lysolipids on the spontaneous curvature and 
bending elasticity of phospholipid membranes., Biophys. J. 81 (2001) 243-254. doi:10.1016/S0006-3495(01)75695-0. 